\title{
WestVirginiaUniversity
}

THE RESEARCH REPOSITORY @ WVU

Graduate Theses, Dissertations, and Problem Reports

2019

\section{Virtual Morphology as a Method of Robotic Control}

\author{
Conner Todd Castle \\ West Virginia University, ctcastle@mix.wvu.edu
}

Follow this and additional works at: https://researchrepository.wvu.edu/etd

Part of the Computer-Aided Engineering and Design Commons, Other Mechanical Engineering

Commons, and the Robotics Commons

\section{Recommended Citation}

Castle, Conner Todd, "Virtual Morphology as a Method of Robotic Control" (2019). Graduate Theses, Dissertations, and Problem Reports. 7418.

https://researchrepository.wvu.edu/etd/7418

This Thesis is protected by copyright and/or related rights. It has been brought to you by the The Research Repository @ WVU with permission from the rights-holder(s). You are free to use this Thesis in any way that is permitted by the copyright and related rights legislation that applies to your use. For other uses you must obtain permission from the rights-holder(s) directly, unless additional rights are indicated by a Creative Commons license in the record and/ or on the work itself. This Thesis has been accepted for inclusion in WVU Graduate Theses, Dissertations, and Problem Reports collection by an authorized administrator of The Research Repository @ WVU. For more information, please contact researchrepository@mail.wvu.edu. 


\title{
Virtual Morphology as a Method of Robotic Control
}

\author{
Conner Castle
}

Thesis submitted to the Statler College of Engineering and Mineral Resources at West Virginia University

in partial fulfillment of the requirements

for the degree of

Masters of Science

in

Mechanical Engineering

Yu Gu, PhD, Committee Chairperson

Jason Gross, PhD

Guilherme Periera, PhD

Mechanical and Aerospace Engineering

Morgantown, West Virginia

2019

Keywords: Robotic Autonomy, Morphological Computation

Copyright 2019, Conner Castle 


\section{Abstract \\ VIRTUAL MORPHOLOGY AS A METHOD OF ROBOTIC CONTROL}

\section{Conner Castle}

This thesis presents Virtual Morphology (VM), a method that explores a different perspective on the design of robot autonomy using inspiration from morphological computing and programmed computation. Morphological computation offers physical solutions that solve complex tasks, like robotic grasping of unknown objects, with relative ease. Unfortunately, these physical solutions are difficult to adjust post-development, and are usually designed to complete only one or a few specific tasks. Programmed computational approaches are more flexible because they can be implemented and adjusted through software, but unfortunately, these approaches can become rather complex as tasks become more difficult. This thesis explores the potential of mixing these methods by implementing morphologic computation, virtually, to couple it with programmed methods; It is implemented through an existing medium of robotics work defined here as artificial physics (AP). The blending occurs by allowing physics simulation to control a physical robot from the interactions produced in the virtual space. The virtual space is projected onto the physical environment to provide robot designers an intuitive understanding of how the interactions dictate the behavior of the physical robot. By utilizing different virtual morphologies, the behavior of the robot can be altered, intuitively. Several case studies demonstrate the capability of VM to generate autonomous behavior. The experiments show that VM can successfully fuse several benefits of morphological computing, such as productive use of passive environmental interactions, with several benefits of programmed autonomy, such as the implementation of high-level goals and quickly changing parameters to adapt for different situations. 


\section{ACKNOWLEDGMENTS}

This research was made possible by NASA West Virginia Space Grant Consortium (Training Grant \#NNX15AI01H) and also from the support provided by the West Virginia Higher Education Policy Commission (award number: HEPC.dsr.18.5). I would also like to thank Henry Vos, Jenn Nguyen, Chizhao Yang, and Scott Harper for their help with this research. 


\section{TABLE OF CONTENTS}

LIST OF FIGURES vii

1 Introduction $\quad 1$

1.1 Problem Statement . . . . . . . . . . . . . . . . 6

2 Literature Review $\quad 9$

2.1 Morphological Computing ..................... 9

2.2 Digital Programming Autonomy . . . . . . . . . . . . . . . . 14

2.3 Artificial Physics-Based Autonomy _. . . . . . . . . . . . . . . 15

2.4 High Level Planning . . . . . . . . . . . . . . . . . . 16

3 Virtual Morphology Design $\quad 18$

3.1 World Environment . . . . . . . . . . . . . . . . . 21

3.2 Physical Robot . . . . . . . . . . . . . . . . . 24

iv 
3.3 Virtual Robot . . . . . . . . . . . . . . . . . . . . . . 25

3.4 Control Architecture . . . . . . . . . . . . . . . . . . 26

$3.4 .1 \quad$ Physical Layer . . . . . . . . . . . . . . . . . . . . . 27

3.4.2 Virtual Layer . . . . . . . . . . . . . . . . . . . . . . . . . . . 29

4 Experimental Design $\quad 33$

4.1 Experiment 1A: Obstacle Avoidance And Goal Reaching . . . . . . . . . . . . 33

4.2 Experiment 1B: Changing The Higher Level Planner . . . . . . . . . . . . . 36

4.3 Experiment 1C: Adding Un-mapped Obstacle . . . . . . . . . . . . . . . . . 39

4.4 Experiment 2A: Orbiting Object With Fixed Circular Path $\ldots \ldots$. . . . . . 40

4.5 Experiment 2B: Dealing With Obstacles While Orbiting . . . . . . . . . . 42

4.6 Experiment 2C: Adding Motion To The Orbited Object . . . . . . . . . . . . . 45

5 Discussion $\quad 47$

5.1 Case Study 1: Obstacle Avoidance and High level Goal Reaching . . . . . . . 47

5.2 Case Study 2: Orbiting Mechanism . . . . . . . . . . . . . . . . . . . 49 
6 Conclusions

Bibliography 


\section{LIST OF FIGURES}

1.1 Virtual Morphology is explored as a middle ground between programming and morphologic methods. . . . . . . . . . . . . . . . . 3

1.2 1.) Positions are initialized. 2.) Virtual force is applied to move towards the goal location (the green point). 3.) Physical body moves towards the position of the virtual body, the virtual body presses against obstacle creating reactionary forces. 4.) The next step of the physics simulation shows the overall movement of the physical and virtual bodies from the combined applied and reactive forces which acted on the virtual body. 5.) Cycle continues as the physical body moves towards the virtual body's position. . . . . . . . . . . . . . . . . 4

2.1 Grabber mechanism from $[$ Gu et al., 2018] . . . . . . . . . . . . . . 10

3.1 Flowchart of the generalized communication scheme. . . . . . . . . . . . 20

3.2 World Environment exists on the air hockey table and includes the Vicon system, the projector, and both the physical and virtual worlds. . . . . . . . . . . 21

3.3 Diagram describing the variables needed to map the virtual space to the real space and showing the coordinate system for both the virtual space and the real space. The depicted triangle is a virtual body existing in the virtual space. . . . . . . . . 23 
3.4 Turtlebot 3 with a base modified with a holonomic drive train . . . . . . . . . . . 24

3.5 Some of the joints available from LiquidFun. The pictures of the joints are from

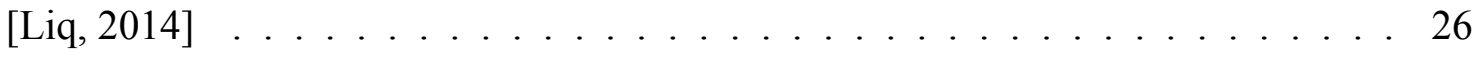

3.6 If the distance between the physical robot position and the virtual robot position increases, a command velocity is sent to the motors in the direction that will minimize this distance. . . . . . . . . . . . . . . . . . 28

3.7 Figure showing the application of applied and environmental forces. Typically, applied forces will act through the center of mass of the virtual body while environmental forces will occur where there was contact with a virtual object. The applied force in this case is acting towards the next node PRM path plan. . . . . . . 30

3.8 Figure showing example of internal and external forces generated in virtual mechanism

4.1 The overhead view of the virtual/physical environment. The example shown is the obstacle avoidance and goal reaching test case. . . . . . . . . . . . . . 34

4.2 Simulation results of the obstacle avoidance and goal reaching test case. Only the simulated trajectories are shown to reduce clutter in the figure, but the physical robot trajectories follow closely. . . . . . . . . . . . . . . 35 
4.3 Simulation results of the PRM method implemented with a solid virtual body. The goal location is the top right of the figure and the starting location is the bottom right. The same PRM graph was used for every experiment in case study 1 . The rigid body was unable to move through the narrow corridor on the right side of the

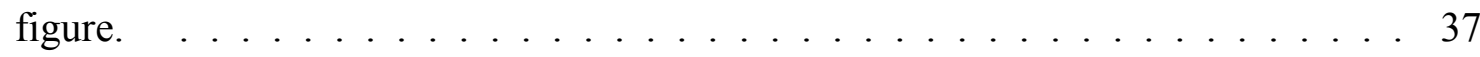

4.4 Simulation results of the PRM path plan with an elastic virtual body . . . . . . . 38

4.5 Simulation results of the PRM path plan with a rigid virtual body and an un-mapped obstacle. The un-mapped obstacle is shown in light blue in the middle of the figure. 40

4.6 Visual representation of the designed orbiting mechanism . . . . . . . . . . . 41

4.7 Orbit of an object using a virtual mechanism to produce trajectory . . . . . . . . 42

4.8 Compliant mechanism designed to handle obstacles presented to the orbit path. . . 43

4.9 The flexibility of the lever joint allows for compliance in the presence of obstacles, in this case the virtual structure collides with the world boundary and therefore bends to continue its orbit. . . . . . . . . . . . . . . . . 44

4.10 A demonstration that the orbit does not need to be about a stationary object . . . 45 


\title{
LIST OF SYMBOLS/NOMENCLATURE
}

\author{
APF Artificial Potential Field \\ IMU Inertial Measurement Unit \\ PRM Probabilistic Roadmap \\ RRT Rapidly exploring Random Tree \\ VF Vector Field \\ VFH Vector Field Histogram \\ VM Virtual Morphology
}

WVUIRL West Virginia University Interactive Robotics Laboratory 


\section{CHAPTER 1 Introduction}

Morphological computing has been recognized in the robotics research community as a way to outsource a portion of a robot's computations to its body [Müller and Hoffmann, 2017]. The physical embodiment of a robot, if properly designed, may allow direct sensing, decision making, actuation, and manipulation when interacting with the environment. Morphological computing has been used as an approach to tackle several robotic challenges in current research such as bipedal walking and self-balancing [McGeer, 1990], and grasping unfamiliar, unwieldy objects [Amend et al., 2012]; even when those objects are located in unstructured environments [Gu et al., 2018]. The noteworthy success of this method is arguably due to the use of clever mechanism designs to achieve tasks through passive interactions with a given environment. Unfortunately, the implementation of these methods in real world problems has proven difficult when those tasks also require deliberate planning and control of the robot, and when the system is used in an environment it was not explicitly designed for. Most of the morphologic mechanisms at play are purpose built to achieve a particular result, and to change the task would mean redesigning and rebuilding the robot. Therefore, there is significant difficulty when attempting to utilize morphological computing in a general purpose sense.

Differing from the morphologic (analog) approach is the programmed (digital) approach. The programming approach here refers to any method by which a digital computer is used to exert control of a robot. This traditionally includes methods for task planning (such as Finite State Machines), techniques for robot motion planning (e.g. D* Lite and RRT* planning algorithms and Vector Field Histograms for obstacle avoidance [Stentz et al., 1995, Karaman and Frazzoli, 2011, 
Borenstein and Koren, 1991]), and other techniques for decision making (e.g., Markov Decision Process [Puterman, 1990], etc.). Typically the digital method relies on the development of digital filters to help process incoming sensory data, digital processing, and computation to turn that input into information that can give the robot some sense of "insight" to its current state and the state of the world around it. Afterwards, algorithmic planning is typically used to determine the robot's next action. These programmed methods typically allow for faster development, prototyping, and tuning of the overall system because it involves changing software rather than physical hardware. The programming-based methods are typically applied to a general-purpose robot and each actuation component is usually actively driven through internal motion components (e.g. motors, linear actuators, etc.). This is opposed to morphological computing, which relies on a task-specific form of embodiment and mechanisms that are commonly passively driven through external interactions. The digital method, obviously, requires the use of a digital computer. The digital computers on board these robots are responsible for the data collection, processing, computation, planning, and output signal generation. While this programmed approach can usually handle more general case problems more robustly than the task specific morphological methods, their is a drawback. As tasks become more complex, the complexity and cost of the computation of the programmed solution also increases significantly. Furthermore, due to a lack of rich physical interactions tailored toward problem solving, as in morphological computation, these programming-based robots are often not able to fully cope with surprises imposed by uncertain environments. The aim of this work is to explore the creation of a method that combines this idea of passive interactions of a body with the environment that was inspired by morphological computing, with the flexibility and ease of adjustment of (digital) programmed autonomy methods. This leads to a differing way of enabling robust behaviors and intuitive control of an autonomous robot. A key hypothesis that is tested during this project is that morphology does not have to be physical in order to realize these beneficial passive behaviors. If these passive behaviors can be reproduced virtually, then they can still be useful in terms of reducing the complexity of generating desirable behavior. Similar to other work in robotic 
control, the backbone for this combination of methods will rely on the use of "artificial-physics" (AP) or simulated physics. This AP is used as the means of producing the passive behaviors from virtual mechanisms. The proposed combination is referred to as Virtual Morphology (VM) and it explores a middle ground between digital and analog robotic computation for enabling robot autonomy and is illustrated in Figure 1.1.

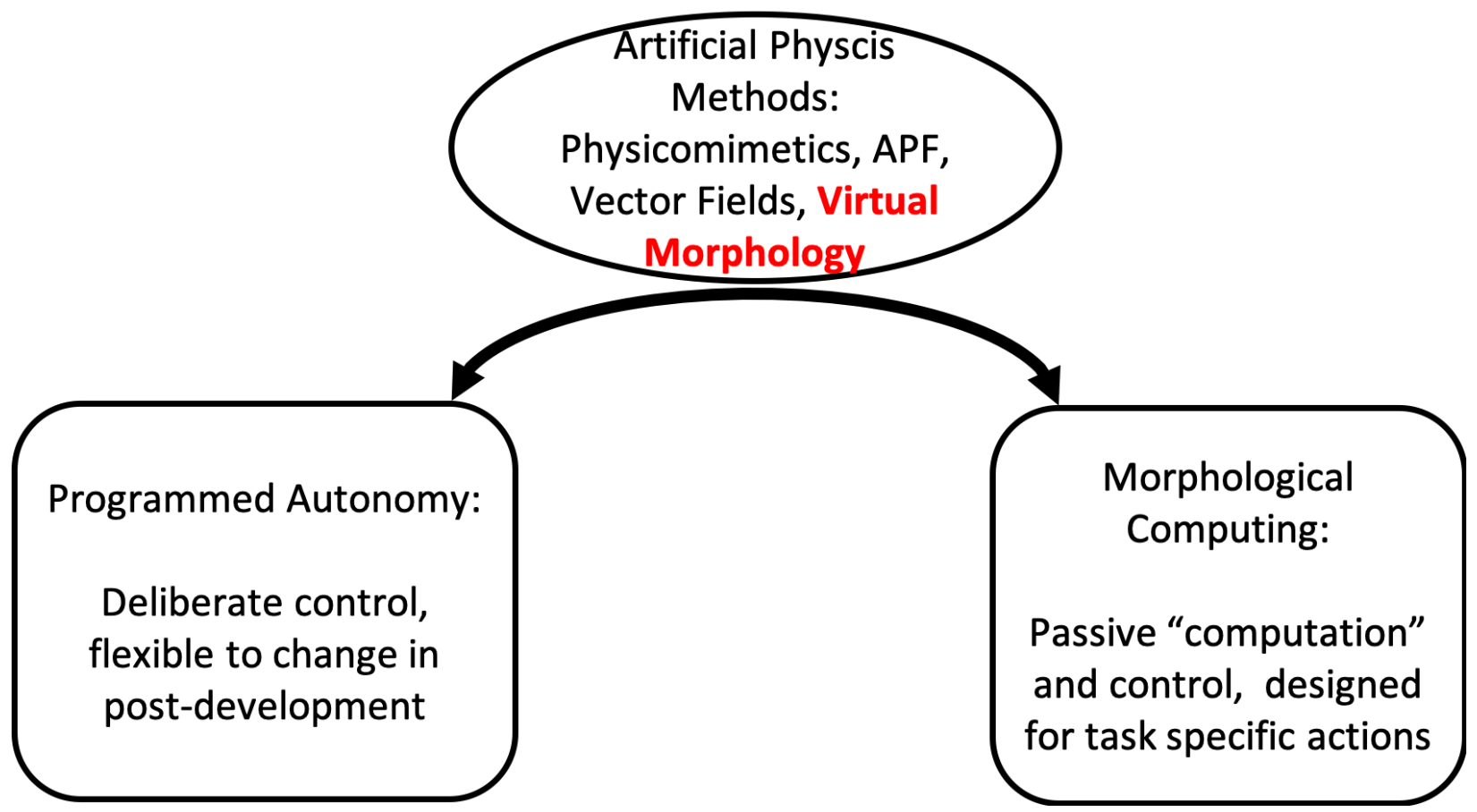

Figure 1.1: Virtual Morphology is explored as a middle ground between programming and morphologic methods.

In VM, a robot would have both a physical body and virtual body. The virtual body performs a similar role as if the physical body were purposefully designed for a specific task. It interacts with a virtual environment through "simulated physics", which then creates virtual forces that induce motion in the virtual body; This is then transformed into motion commands for the physical body. More specifically, control commands are computed and sent to the physical body to match the position and orientation of a given attachment point on the virtual body. Figure 1.2 shows a visualization of this control process. The passive virtual body "controls" the actions of the physical 


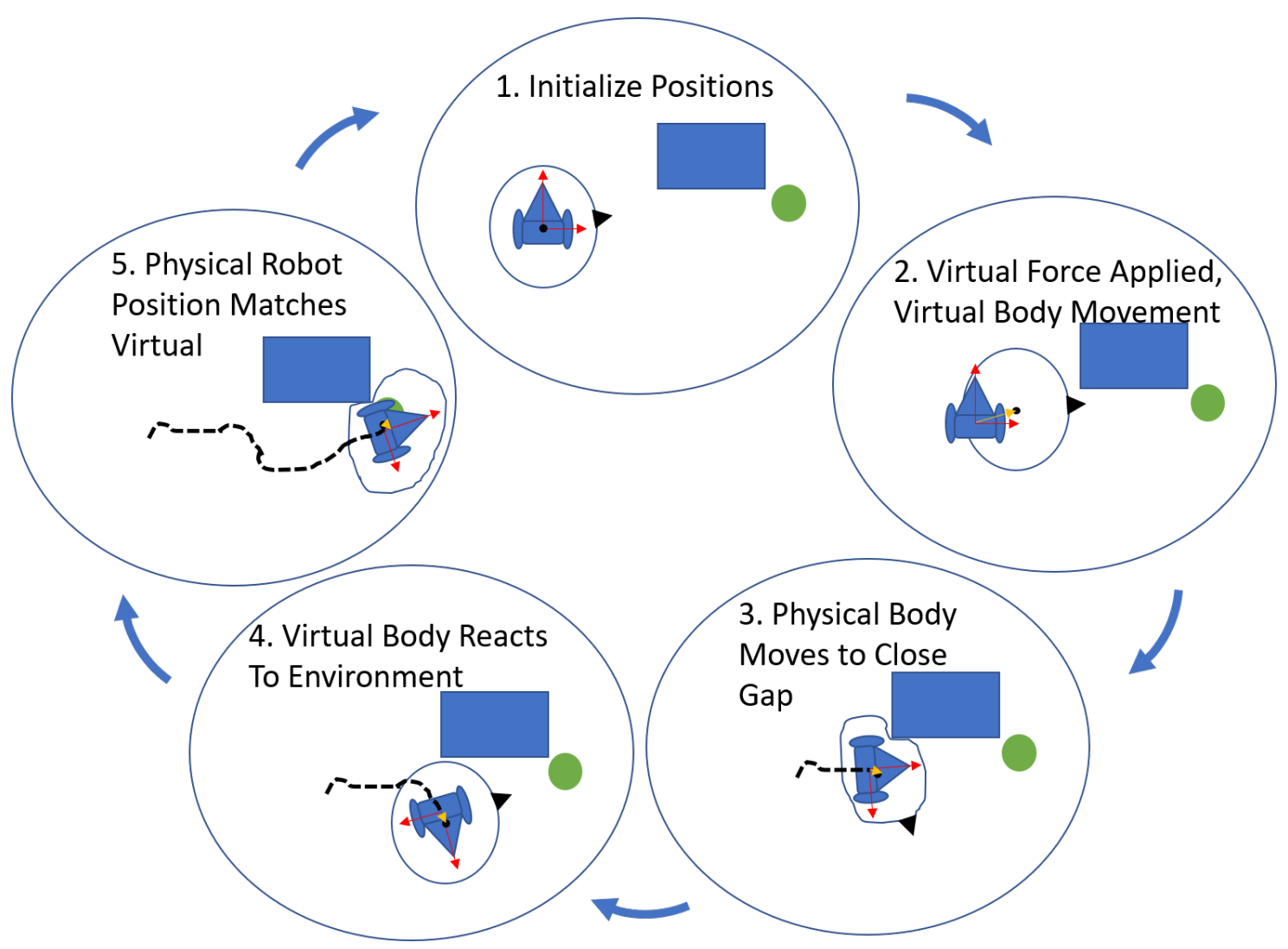

Figure 1.2: 1.) Positions are initialized. 2.) Virtual force is applied to move towards the goal location (the green point). 3.) Physical body moves towards the position of the virtual body, the virtual body presses against obstacle creating reactionary forces. 4.) The next step of the physics simulation shows the overall movement of the physical and virtual bodies from the combined applied and reactive forces which acted on the virtual body. 5.) Cycle continues as the physical body moves towards the virtual body's position.

robot. In this manner, the physical body is allowed to be entirely actively controlled, as in, a digital computer controls every output of the physical robot (i.e., drive motors), while the virtual body is passively driven, as in, control is dictated by the virtual forces applied to its body. The hope of utilizing both passive and active components in the control structure is to achieve some of the benefits of morphology discussed previously while maintaining full digital control over the robot.

Through the introduction of the VM approach for robot control, this thesis claims the following contributions: 
1. VM provides a framework for infusing some of the benefits of morphological computing (e.g. robustness to uncertainties) into a programming-based system potentially allowing it to meet a variety of high-level objectives, achieve a higher level of generality, and allow it to be more easily tunable after design of the system. This method can adapt to different tasks on the fly by changing virtual bodies, or by changing the higher level software, which allows for fast redesign/evolution. It also has no dimension, weight, and cost limitations associated with physical morphological designs;

2. VM allows easy visualization of the virtual body and how it interacts with the environment. This can support the development of intuitive methods for designing and tuning of the robot behavior by changing the robot virtual morphology;

3. The effectiveness of VM in solving real robotics application problems are demonstrated through several experimental case studies in this project.

The proposed VM approach, in the current form, also has several limitations. First, VM consumes substantial computer resources (i.e., relies on a physics engine instead of analog morphological computing). Second, the lack of physical embodiment of the virtual body means no easy way of manipulating the environment; and the feedback received from the virtual interactions may not be as reliable as the results from physical interactions. Lastly, the capabilities of the robot control and behavior generation is limited to the capabilities of the simulated physics; therefore if some property is not included in the physics simulation, it cannot be reliably reproduced in the physical robotic control.

The development of VM is considered as a first step towards a long-term vision: allow robots to balance the computation performed by the digital computer, through deliberate reasoning and virtual body simulation, and by morphological computing, through the physical body-environment 
interactions. This trade may be shifted online as different task and environmental requirements arise. Some ideas towards realizing this vision will be briefly discussed at the end of this thesis in a future work section.

\subsection{Problem Statement}

The objectives of this thesis are to:

1. Determine which, if any, of the principles of physical morphology can be utilized virtually to enable behavioral control of the robot.

2. Build a system to demonstrate and implement the blending of those principles with digital methods.

3. Explore the design of several use-case experiments of this method to determine its efficacy to solve a given problem.

Two case studies were conceptualized in order to demonstrate the VM design process and its benefits. The first case study is about enabling basic robot autonomy through the demonstration of obstacle avoidance and goal reaching behaviors. The objectives of this case study are to show that: 1) the behavior of a VM controlled physical robot is easily adjustable by changing the virtual "mechanical" properties, such as shapes and material properties, of the virtual body; 2) there is a natural way of integrating multiple robot behaviors with the proposed approach. For example, combining VM based obstacle avoidance with traditionally programming based motion planning and control methods. The second case study is about solving a non-trivial motion planning problem: controlling a robot to orbit an object to perform an inspection task. The objectives of this 
case study are: 1) demonstrate that the mapping of a complex programming problem into a different design space (i.e., trajectory planning problem transformed into a mechanism design problem) could significantly improve the intuition of the robot control process; 2) demonstrate that new robot behaviors can be quickly enabled by exploring small variations of VM designs.

It should be specifically noted that the case studies presented in this project have been successfully completed by multiple researchers using other various methods. Examples include that of [Kapitanyuk et al., 2017] and [Pereira et al., 2016] who have found solutions for similar orbiting/ path following tasks as case study 2, and likewise [Quinlan and Khatib, 1993] completed a method capable of solving case study 1. This project does not claim to solve a specific novel problem, incapable of being solved by any other method, or that it will solve the problem better than another method. Rather, the hope is that by tackling known problems from a different perspective may provide some helpful insight to designing autonomy from a different direction. Therefore the VM method, in this thesis, is not compared to existing methods in terms of performance but rather is compared by its own ability to complete the problem at hand, and will be observed for any benefits or disadvantages that arise from tackling the problems from this differing perspective.

Throughout this thesis, it is assumed that the pose of the robot is known with a high-accuracy, the robot can fully sense its local environment, and the physical robot has a holonomic drive-train and sufficiently fast dynamics so it can be used to respond to the movement of the virtual robot body. It is also assumed that while the robot has access to a global map, the global map may include inconsistencies and also errors in terms of the number and location of obstacles.

The rest of this thesis will be organized as follows. First a discussion of background research is presented in order to provide context for the motivations behind the VM method and why it was developed in this manner. Next a detailed report of the design of the VM method is shown 
to give the reader the understanding of the inner workings of what was needed to create VM, and provide enough insight to recreate the method if so desired. This includes a discussion of the world environment, both virtual and physical, the physical robot, the virtual embodiment, the control architecture, and also how all of the individual components communicate with one another. Next the design of the experiments developed for each case study is discussed in detail along with the results of each experiment and any important observations discovered along the way. Finally, the overall results of the project, concluding remarks, and future directions of the work are discussed. 


\section{CHAPTER 2 Literature Review}

For organizational purposes, the literature review has been broken into subsections as follows: morphological computing, digital programming autonomy, artificial physics based autonomy, and high level planning.

\subsection{Morphological Computing}

In order to blend morphology with programmed methods for behavior and autonomy design, it is important to understand some of the basic driving factors behind morphological computing. In other words, what aspects of morphology are important to consider, or to imitate, when attempting to reproduce it virtually with VM? After careful study, two main drivers were observed in morphology that were considered beneficial, and able to be reproduced virtually to achieve a desired result. These drivers were as follows: some sort of embodiment is required with which to interact with an environment, and physics should dictate interactions with that body and its environment. By leveraging these driving factors, researchers have been able to produce desirable autonomous behavior through the use of designed morphological mechanisms. It is important to note that the second factor needs to drive the first. In other words, the embodiment should be interacted with by an external force, and then that force will cause passive elements of the embodiment to actuate in the desired manner that the designer intended. This is important because this passiveness is what allows the system to be driven without active input, for instance you wouldn't need a digital controller telling the system when to actuate. It is also a way to allow the system to handle in- 
consistencies in the environment. The external force can be thought of as the actuation force and the embodiment would be considered the controller because it turns this driving force into motions desirable to achieve the overall task. While the mechanisms may not be intelligent, they can be thought of as autonomous, or performing tasks on their own with little to no user input.

The observations stated in the above paragraph are shown in practice by the examples below. The bipedal walker from [McGeer, 1990] is able to balance itself and walk passively down a slope. Gravity is the power for the robot, and the cleverly designed walking mechanism (i.e., the physical embodiment) is the motion controller; the operator only needs to "initialize" the system by placing the robot on the slope. Essentially the external force of gravity, starts a mechanism motion which is designed to catch itself as it falls. After it catches itself, the process repeats on the next leg and continues to cycle until the gravity force is perpendicular to the legs. The external force is transformed into internal forces due to clever mechanism design and allows it to cycle in a controlled manner. The robot unfortunately can't turn or stop on its own, but the walking task that it was designed for, it can complete flawlessly.
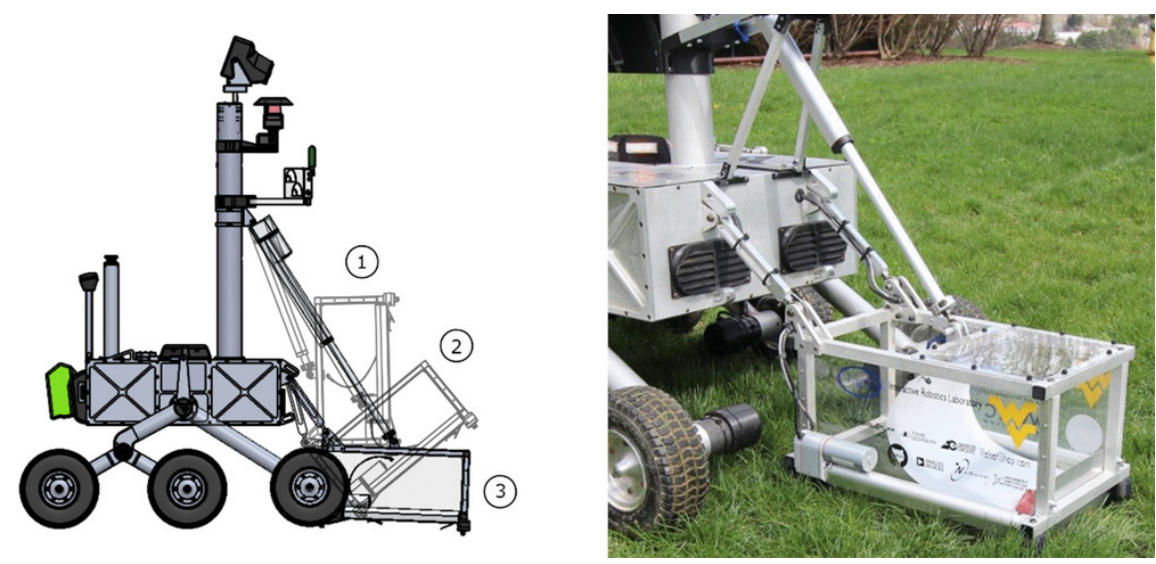

Figure 2.1: Grabber mechanism from [Gu et al., 2018]

Another example of this demonstrated advantageous passive behavior is exhibited through the grabber mechanism from [Gu et al., 2018]. This grabber was designed to be purposefully under- 
actuated. That is, it has one actuation element centered on top of the grabber to lower it to the ground, and two hinge joints which are attached to levers that mount the grabber to the rest of the body. In this configuration, the grabber "forms" more flexibly to the terrain, especially in the presence of slopes, than it would have if the box was lowered horizontally relative to the body with a more rigid mechanism. This allowed more consistency in obtaining samples than previous iterations of the grabber. Another way to achieve this compliance is through the use of a fully actuated gripper (i.e., the full pose can be digitally controlled and actuated). Unfortunately this would require some way to obtain the terrain slope digitally so that the computer could then compute the angles needed to tell the gripper how to orient itself to match the slope of the terrain. Morphologic computation researchers argue that the passive compliance of the under-actuated design is a form of computation, offloaded to the physical mechanism. Therefore, with the under-actuated design, the robot's computer does not need to calculate the slope and then match it with a fully active grabber, it only needs to actuate to box downwards to the ground and the morphology of the grabber will handle the rest.

Along the same vein of grasping tasks is the granular gripper from [Amend et al., 2012]. The granular gripper is a universal gripping mechanism that relies on a physics phenomena known as particle jamming. When the gripper touches an object, the particulate matter inside the gripper gives way as the object "pushes" back against the gripper. This causes the gripping mechanism to passively mold to the object. Then a vacuum pump is switched on and pumps the air away from the gripper, effectively locking the particulate in place. With the particulate locked around the specific shape and curves of the object, the object effectively becomes attached in place to the gripper. This allows the gripper to pick up virtually anything. This is a great example of the principle of using passively generated forces to the advantage of the user. By taking advantage of natural reactionary forces placed on the gripper, the gripper morphs to the object and creates a better grasp than could be generated if the particulate did not give way to the object. This gripper, the walker, and all the 
examples given so far of morphological computation show this same trend of a body being acted upon by physics, where external forces generate a desired movement of the body because of its specific design. It is clear that changing the mechanism, the environment, or the forces can change the behavior.

Morphology such as these examples typically takes place under specific circumstances which are carefully taken into consideration in the design process. They are purpose-built designs and generally do not extend beyond their own reach. The gripper was designed very well for grasping tasks but what if the task was switched to in-hand manipulation? Without dexterous phalanges, the gripper can no longer perform the task. In order to switch to this task an entirely new gripper would need to be utilized. This leads to some key questions: How can these mechanisms be created reliably? Is there any way to automate the mechanism design so that the operator does not have to continuously create more designs? These questions are important not only to morphology design but also to the VM method; While VM may not rely on physical design changes in the scope of this thesis, it still relies on the creation of different mechanisms, albeit virtual mechanisms, in order to obtain desirable behavior. Being able to generate new designs automatically would also have a particularly unique benefit to VM over physical morphological computing because new "virtual" mechanisms can be implemented immediately into the system to enable new behavior; Which could lead to rapid prototyping and testing of behavioral control, whereas they would still need to be physically attached in physical morphology.

Work from [Greiner, 1990], does an excellent job describing the design methodology of the creation of another passive gripping mechanism relying on morphological principles. The gripping mechanism is designed in such a way that when an object is pressed against the gripper, the gripper begins to close around that object, passively. Usually, the creations of these mechanisms rely on some sort of intuitive understanding from the designer. It is arguable that this intuitive under- 
standing has a low barrier to entry for designers as many people could think a some sort of design that could complete their task. Unfortunately, it still takes much work and much thought to take a designed mechanism and optimize it to meet a desired performance requirement.

To tackle optimizing design parameters, [Ha, 2018] used reinforcement learning techniques. In their method, a designer creates the mechanism at a high level, deciding on the bodies, joint placement, etc.. Then the designer would let a reinforcement algorithm process determine the physical details such as size and length of the bodies in order to achieve a more optimal design based on desired parameters. This allows the designer to achieve an overall desired mechanism without the considerable work needed to tune the mechanism design as that portion can be performed automatically.

Some researchers have tackled the task of autonomous mechanism design as a whole, from the standpoint of evolutionary algorithms, with significant success. For instance, [Sims, 1994] evolved several different locomoting simulations using a body generation strategy. Also, [Cheney et al., 2014] expanded from this work by including multiple different types of materials to be mixed into another body generation scheme to come up more complex bodies and locomotions. By adding and distinguishing between hard, elastic, actuating, and non-actuating body components they were able to show bodies that were evolved to achieve specific advantages in a given environment. Another work from [Lipson and Pollack, 2000], not only automatically generates these bodies but then also attempted to realize them in the physical world through rapid prototyping techniques.

Although automatic optimization and generation of mechanisms is out of scope for this thesis work, it provides at least two useful insights.

1. By studying mechanism design, one becomes better acquainted with how to achieve desired 
motions through the use of mechanisms, and types of common motions that already have pre-made common mechanisms associated with them. In this way the designer has a larger set of mechanisms or "tools" they may pull from to accomplish tasks that may be a fairly typical desired motion.

2. It allows for a starting point for future work in which a robot may decide for itself, what mechanism is best suited for a particular behavior it needs.

\subsection{Digital Programming Autonomy}

Contrary to the passive, task specific nature of morphologic computation, digital methods are usually performed on more general purpose systems. Instead of designing a gripper that molds to the object, one would design an algorithm to open a general purpose robotic gripper with multiple "fingers" and choose how tightly to grab and where to grab on an object. The morphologic process of the granular gripper can handle inconsistencies because the particles will form around an object passively and do not need to know what the object looks like. The granular gripper doesn't need a digital model of the object because it already has the perfect model to reference, that is, the object itself. Whereas when grasping with a dexterous, fingered manipulator the size, geometry, weight, roughness, etc. all become much more important factors because these variables factor into the calculations of how best to open and close the gripper around the object. Typically, these general purpose grippers cannot obtain the rich information that comes naturally from the morphologic process. While there is significantly more complexity and computation involved in this programmed process, it also allows for very complex and general actions to occur on a variety of objects. For instance picking up and the rotating an object in hand. [Akkaya et al., 2019] recently was able to successfully manipulate a rubix cube in hand using digital autonomy methods, however they rely on artificial intelligence in order to accomplish the manipulation tasks. This speaks to the complexity 
of using a general purpose gripper with programmed methods and why so frequently researchers seek out the benefits of morphology to help make tasks easier.

The VM approach hopes to blend benefits of morphology with digital autonomy in such a way as to create autonomous behaviors from a different perspective. That is, it hopes to recreate a virtual version of the analog computations that naturally occur through physical morphologic computation, and potentially alleviate some of these complex computations present in general-purpose robotic systems. To accomplish this in a digital architecture, in this case, requires the use of artificial physics.

\subsection{Artificial Physics-Based Autonomy}

In order to properly utilize the discussed drivers of morphologic computation, VM relies heavily on physics. Instead of using actual physics as in the case of physical morphology, it will rely on physics emulation. Using physics inspired control is not a new subject in robotics. Much research has been conducted that uses the inspiration of physics principles for control. These methods of artificial-physics (AP) control include vector fields (VF), artificial potential fields (APF), and physicomimetics. Vector fields may not technically consider artificial physics principles in the same manner as physicomimetics or APF, which are directly inspired by physics properties; but they do share similar principles of generating movement controls, and as such, are considered to be related to the broader class of AP methods in this thesis. These AP approaches have tackled problems concerning path planning, trajectory generation, obstacle avoidance, and control for collaborative multi-robot systems and swarm robots. APF and VF are typically used for solving path planning problems with inherent obstacle avoidance. Based off of original work from [Khatib, 1985], both [Lee and Park, 2003], and [Warren, 1989] use APF methods to solve goal 
reaching tasks while also avoiding obstacles. Similar work has been performed utilizing VF methods. [Apker and Potter, 2012], and [Spears et al., 2004] have taken motivations from AP methods and extended it into use for formation control of multi-robot and swarm robot systems. For instance they used liquid and gaseous physics motivations to create controllers for swarm robotics. They successfully ran simulations to control many robots, all performing different motion plans, simultaneously with a controller that dictated individual and bulk motions. While Spears worked with holonomic point particles, Apker was able to extend this into the utilization of a virtual "body" to determine control input for a differential drive robot. Virtual embodiment was also utilized in [Liu et al., 2018] through the the creation of a virtual "linkage". Essentially Liu's linkage is a deformable virtual member which allowed a formation of robots to deform in an environment. This is largely beneficial because it allows for compliance to a given environment. Liu used this method to rotate a grouping of robots around a point, and also used it to deform a formation through a narrow corridor while maintaining constant spacing of the robots. The robots were attached to the linkage at evenly spaced intervals. Particularly present in the work from Liu, Lee, and Apker is the addition of a more rich virtual representation of the body. This leads to the thought process that virtual embodiment beyond the point particle allows for a more rich motion control to be developed, allowing for control of a more complex physical robot. Similarly, the VM method serves to explore the possibility of this thought further by expanding the use cases and the type of emulated physics employed to determine if the use of virtual embodiment can create complex desirable behaviors.

\subsection{High Level Planning}

While the previous methods describe ways to implement various AP methods as full solutions to path planning problems, the VM method will utilize AP as a method of obtaining behaviors from governing physics principles. Therefore when VM is implemented with a full path plan, AP 
may not necessarily act as the whole planner but might only act as a reactive element in a larger planning system. [Pereira et al., 2016] implemented a similar approach where there is a VF global layer and an optimal planning low level layer, combined to achieve desirable properties of both methods. They were able to successfully utilize VF to create a global trajectory plan, while using

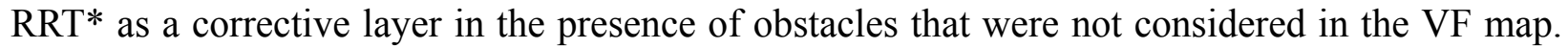
VM implements multi-layered planning structure similar in spirit to [Quinlan and Khatib, 1993]. In [Quinlan and Khatib, 1993], a path is obtained from some initial planner which links a starting location with a goal location. This path is treated as an elastic "band" which is interacted on by artificial forces generated by nearby obstacles which could be detected in real time by on-board sensors. These forces stretch and bend the path until it is collision free, successfully linking a reactive layer into a high level path plan. Instead of using the artificial forces to directly alter the path, VM uses artificial forces to interact with a virtual body that is attempting to follow a path to layer a reactionary behavior into a higher level plan. 


\section{CHAPTER 3 Virtual Morphology Design}

The utilization of VM in this thesis is primarily focused on controlling a physical robot using a virtual embodiment. This method requires a physical robot and environment as well as a virtual robot and environment. This section describes the full system used to create this general form of control. This is not the only way for VM to be implemented but it describes a general purpose implementation of VM for robot behavioral control. The required components for general purpose use of the VM method are listed as follows:

- Virtual Components

- Virtual Description of Embodiment: this can be anything from a point particle to a complex mechanism made of multiple linkages, joints, etc. It serves as the method of obtaining interesting reactive, or passive behaviors;

- Virtual World: the virtual embodiment of the robot needs an environment to interact with and therefore some sort of environment needs modeled as well;

- Physics Emulator: this is used to compute the physics between the virtual embodiment and the virtual world.

- Physical Components

- Physical Robot: A physical robot is the main element to be controlled so it is needed to complete the method. However, much preliminary work can be performed entirely through simulation before it is used to control the physical robot; 
- Localization Method: The physical robot needs a high degree of confidence of its position in the real world to ensure that the behaviors will be generated properly when converting to the virtual world and back again;

- Local Sensing Method: While the physical robot does not need to know the global map in order to generate behaviors, it will need accurate local sensing so that any local objects will be properly mapped into the virtual world.

- Controllers

- Physical Robot Controller: The physical robot needs a controller that minimizes the distance between the virtual body and the physical body. This controller needs both the global pose of the physical robot in real space and the global pose of the virtual body in real space. The global pose of the virtual body in the real space is found from a linear transform from virtual space to real space;

- Virtual Controller: This controller is dictated through a high level planner, if desired, as well as through the physics emulator. It requires knowledge of the high level plan, if a high level plan is implemented, and also the virtual world. The virtual controller is described in subsection 3.4.2.

The general communication between these different components is shown in Figure 3.1. This work specifically uses LiquidFun [Liq, 2014] as the physics engine and the Robotics Operating System (ROS) as the backbone for communication between individual programs. ROS is a common tool used in robotics as a way to connect multiple separate programs together. These programs are referred to as nodes and they share data through data packets known as topics. In this case, ROS is used mainly to bridge communication between the physics engine, the localization method, and the physical robot controller. LiquidFun will be discussed in more detail later in this section. 


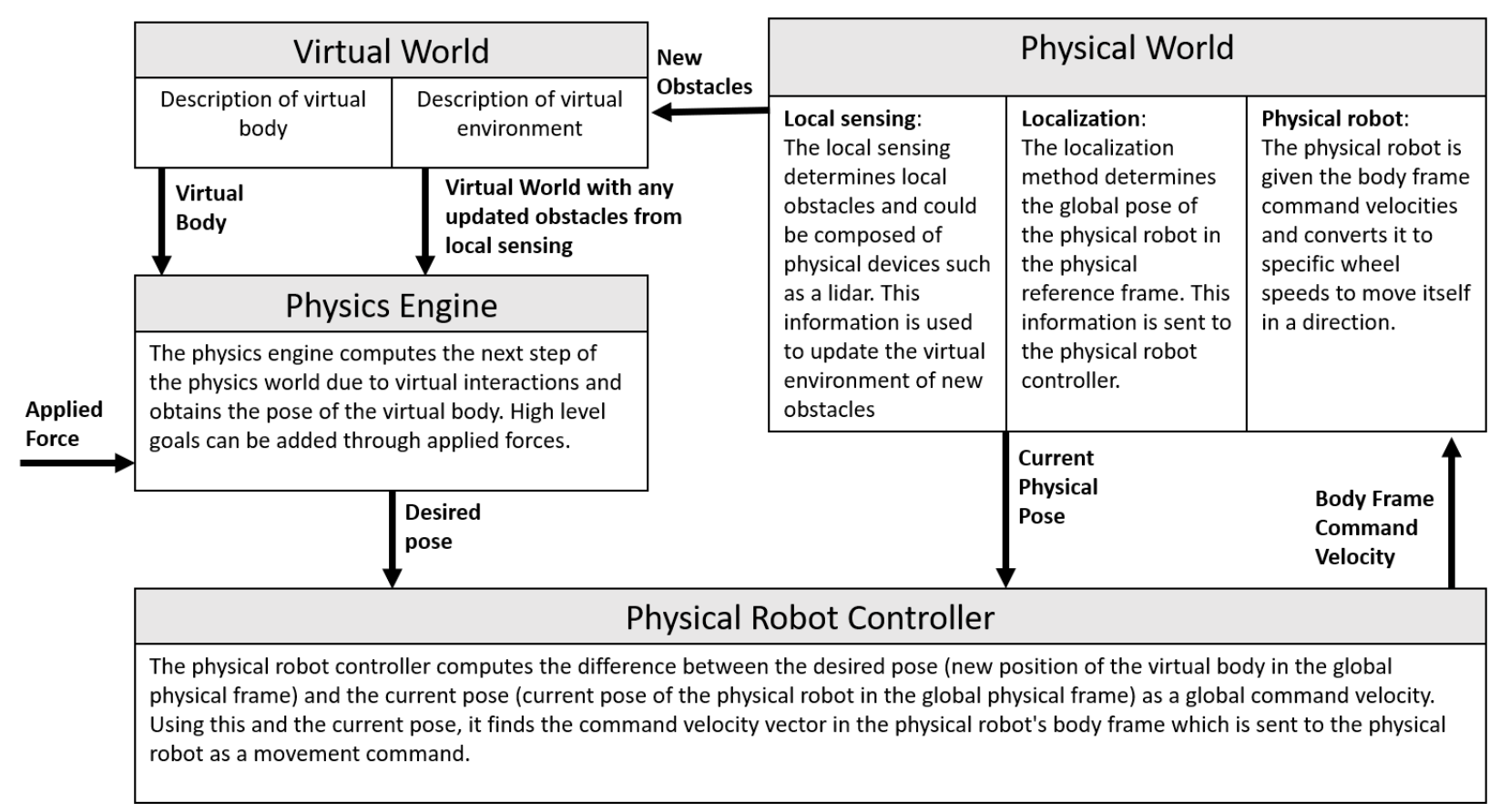

Figure 3.1: Flowchart of the generalized communication scheme.

The organization of the rest of this chapter, which describes the particular implementation (i.e. specific hardware and software used) created during this project, is as follows. First the world environment, including both the physical and virtual environments will be described. Next, a discussion of the physical robot is necessary in order to understand the rest of the system. This will be followed by a discussion of the construction of the virtual embodiment of the robot. This will also include how the physical robot is "attached" to the virtual body. Then a description of the multi-layered control scheme is discussed. Finally, the design of experiments is presented as well as the arguments for why they are good indicators of how the VM method meets the problem statement. 


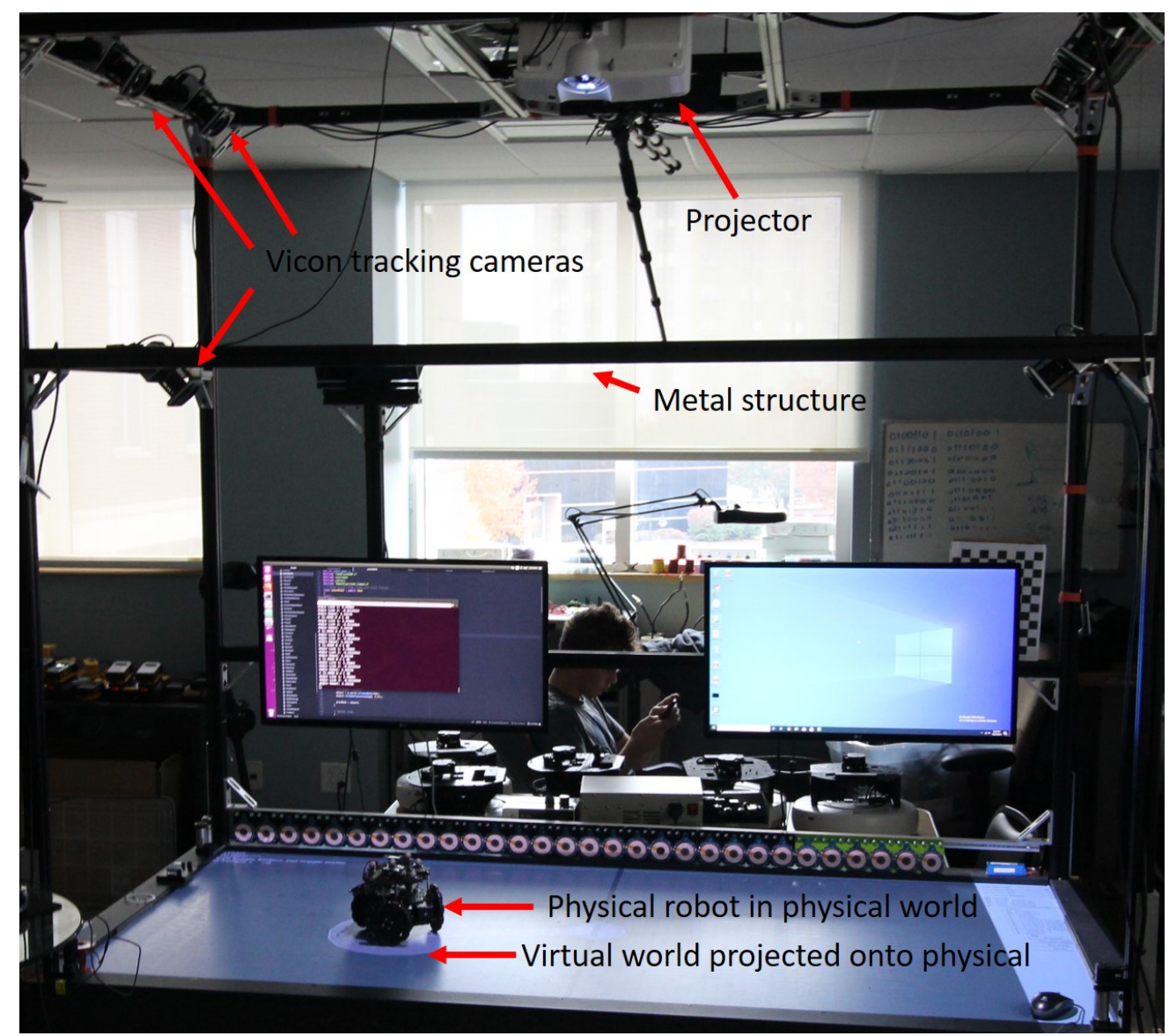

Figure 3.2: World Environment exists on the air hockey table and includes the Vicon system, the projector, and both the physical and virtual worlds.

\subsection{World Environment}

As shown in Figure 3.2, the world environment consists of both the physical and virtual subenvironments. The physical environment is built on top of an air-hockey table with a large, aluminum box structure anchored to its top. This metal structure contains the main tracking and visualization equipment for the project. The localization of the physical robot is given from a Vicon motion tracking system. The Vicon system uses retro-reflective markers placed on top of the robot to track its position. It collects the full 6-DOF pose of the robot in 3D space. There are 6 Vicon cameras attached to the metal frame and a separate computer that runs the Vicon software, which 
is then sent through a local WiFi link to the main computer of the table. The visualization system is a powerful short-throw projector also attached to the top of the metal structure. The projector projects the virtual world on top of the physical so a user can get an idea of what is occurring during real-time operation of the system. The visualization system is not needed to execute the robot, it is only meant as a tool to gather intuition. All that is needed for the system to run is a computer to run physics, localization of the robot in the world environment, local sensing of the obstacles around the robot, and the programs meant to link the virtual and physical worlds. The tabletop edges define the boundary for both the physical and virtual worlds.

As stated previously, the virtual world in this work is constructed from a physics engine known as LiquidFun. This engine was chosen for a few reasons. First, because the VM method is an exploration of what is possible in terms of artificial physics-based control, it was not desirable to program all of the functionality by hand if an existing system already contained desirable physics simulation. Because of the prototype nature of this study, speed and efficiency were not of primary concern, so using an off-the-shelf physics engine was sufficient as opposed to creating one from scratch. Secondly, the engine is a 2D simulation. The early experiments to be modeled all relied on a 2D world representation. There are other 3D physics simulators (e.g. Bullet, Gazebo, V-Rep, etc.) but these only provide $2 \mathrm{D}$ approximations. Since all of the preliminary work was to be done using 2D physics, it was more desirable to use a simulator built on that principle. Finally, it was also chosen for its ability to utilize liquid and particle-based physics. This allows the experiments to benefit from deformable, particulate bodies, which leads to more interesting behaviors. However, the physics system was designed to be replaceable in the event a better solution, or a solution that fits the criteria of the problem was discovered later.

The virtual and physical worlds are mapped together through a linear interpolation between the extreme boundary locations in $\mathrm{x}$ and $\mathrm{y}$. The coordinate frame origins and axes were aligned 


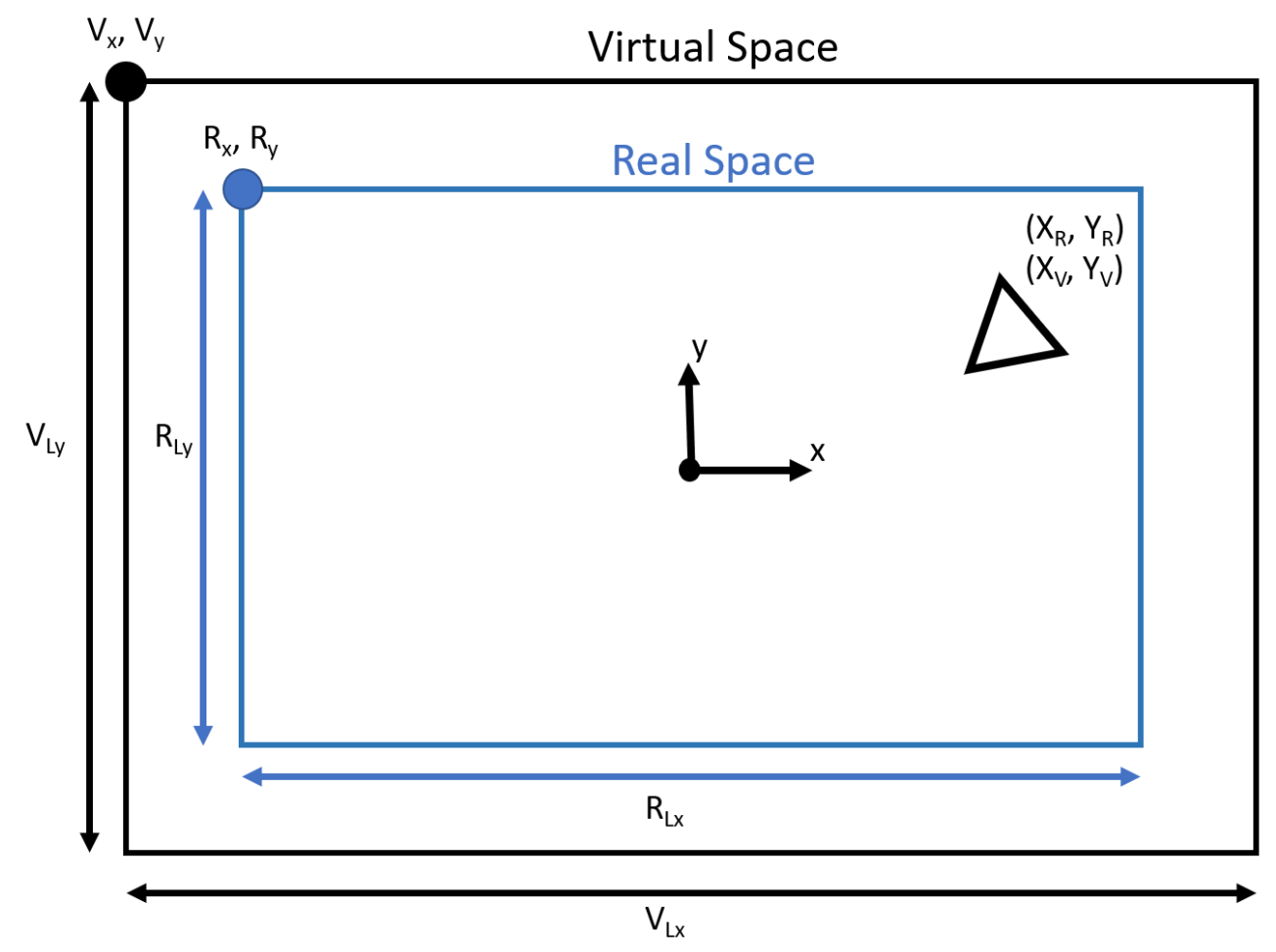

Figure 3.3: Diagram describing the variables needed to map the virtual space to the real space and showing the coordinate system for both the virtual space and the real space. The depicted triangle is a virtual body existing in the virtual space.

together as shown in Figure 3.3. The equations below describe how a known virtual position of a virtual body can be transformed into the real space (i.e. $X_{\text {Real }}^{\vec{a}}=g\left(X_{\text {Virt }}\right)$ ).

$$
\begin{gathered}
X_{R}=\frac{R_{L x}}{V_{L x}}\left(X_{V}-V_{x}\right)+R_{x} \\
Y_{R}=\frac{R_{L y}}{V_{L y}}\left(Y_{V}-V_{y}\right)+R_{y}
\end{gathered}
$$

$\left(X_{R}, Y_{R}\right)$ and $\left(X_{V}, Y_{V}\right)$ are defined as the physical global position, and the virtual global position of the virtual body, respectively. $\left(V_{x}, V_{y}\right)$ is an arbitrary point in the virtual space and $\left(R_{x}, R_{y}\right)$ is a corresponding point in the real space; These points were chosen as both the virtual 
and real origins since they overlapped one another. $V_{L x}, V_{L y}, R_{L x}$, and $R_{L y}$ all correspond to the lengths of each axis, up to the boundaries of the table, of the virtual and real spaces.

\subsection{Physical Robot}

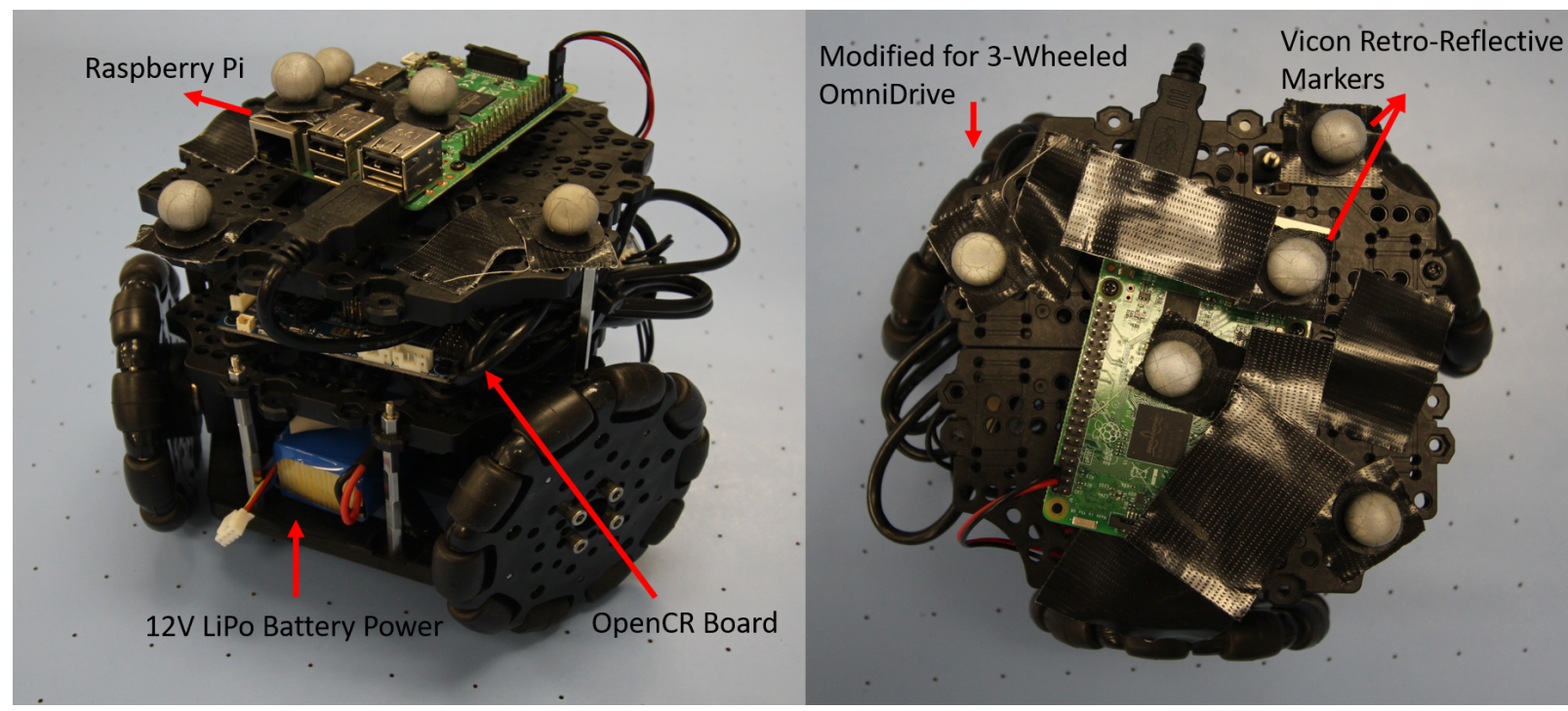

Figure 3.4: Turtlebot3 with a base modified with a holonomic drive train

The physical robot is a modified Turtlebot3 burger model from Robotis [Tur, 2019]. The Turtlebot was, originally, a differential drive robotic system. It relies on a Raspberry Pi as a computational resource, and its OpenCR board to handle low level communication to the on-board sensors and actuators. The actuators were originally two dynamixel motors. The robot was modified to use a holonomic drivetrain to allow the robot to independently rotate and translate, which simplifies planning because there are less motion constraints that need to be considered when determining a valid path. The VM method is not limited to the holonomic drivetrain, but it was deemed sufficient for this early exploration of the method. Three methods of obtaining holonomic drive in a 2D plane are from the 3-wheeled omni-drive, 4-wheeled mecanum, and swerve drive robot configurations. A 3-wheeled omni-drive system was chosen because it allowed for the least modification of the 
physical Turtlebot platform. The kinematic equations for a 3-wheel omni-drive configuration are shown below. This equation was pulled directly from [Lynch and Park, 2017], more information about different drive configurations can be found there.

$$
\left[\begin{array}{l}
u_{1} \\
u_{2} \\
u_{3}
\end{array}\right]=\frac{1}{r}\left[\begin{array}{ccc}
-d & 1 & 0 \\
-d & -\frac{1}{2} & -\sin \frac{\pi}{3} \\
-d & -\frac{1}{2} & \sin \frac{\pi}{3}
\end{array}\right]\left[\begin{array}{l}
\omega_{z} \\
v_{x} \\
v_{y}
\end{array}\right]
$$

$u_{1}, u_{2}$, and $u_{3}$ are the rotational velocities of each drive motor and $\omega_{z}, v_{x}$, and $v_{y}$ are the rotational and translational components of the global velocity vector. $r$ is the radius of the wheels, and $b$ is the distance from each wheel to the center of the robot platform. The wheels are evenly spaced $120^{\circ}$ from each other.

\subsection{Virtual Robot}

The virtual robot is composed of at least a virtual structure. A virtual structure is a body in which the virtual physics engine can act upon. The virtual robot can also be extended into a virtual mechanism by adding connection joints to other structures. These joints can also have attached motors, joint limits, etc. LiquidFun provides a variety of joint types some of which include revolute, prismatic, gear, and distance. Other interesting joints can be found in the LiquidFun programmers guide [Liq, 2014]. A short description of the joint types is given in Figure 3.5. The ability to create a virtual mechanism with structures and joints that are acted upon by physics principles is a core principle that differentiates the VM method from other behavior and planning related tech- 


\begin{tabular}{|l|l|}
\hline \multicolumn{1}{|c|}{ Description } \\
\hline Revolute Joint: & $\begin{array}{l}\text { Causes two virtual bodies to be locked } \\
\text { together by a single hinge point. Bodies } \\
\text { can rotate relative to one another. }\end{array}$ \\
\hline Prismatic Joint: & $\begin{array}{l}\text { Causes two virtual bodies to be } \\
\text { constrained to move relative to one } \\
\text { another through a single vector direction. } \\
\text { The bodies can no longer rotate relative to } \\
\text { one another. }\end{array}$ \\
\hline Gear Joint: & $\begin{array}{l}\text { Connects two joints together (either 2 } \\
\text { prismatic, 2 revolute, or 1 prismatic and 1 } \\
\text { revolute), such that when one moves, the } \\
\text { other moves an amount equal to the gear } \\
\text { ratio multiplied by the movement of the } \\
\text { first. }\end{array}$ \\
\hline
\end{tabular}

Figure 3.5: Some of the joints available from LiquidFun. The pictures of the joints are from [Liq, 2014]

niques. Others have touched on this to some degree, [Liu et al., 2018] created a basic linkage virtual structure for formation control and [Apker and Potter, 2012] shows the use of a virtual "dumbbell" structure to facilitate control for a differential drive robot. However, VM emphasizes the use of virtual structures, joints (linkages), and motors to enhance control of a physical robot to a much higher degree.

\subsection{Control Architecture}

The control scheme of the full robot system can be described as a "leader-follower" pair. Essentially, the physical robot acts as a "follower" to the virtual component. It behaves as a shadowing 
element and therefore will attempt to reproduce any trajectory made by the virtual component. The virtual component dictates the movement of the physical robot in order to take advantage of the "passive" nature of how the virtual body interacts with its virtual world. Because of this, if desired, the virtual component can fully simulate a "session" offline, and could have the physical robot reproduce the trajectory later. In this work, this is not the case. The virtual world is simulated online and the physical robot follows this motion in real-time. This has the particular benefit of allowing the physical robot to inform the virtual world of any un-mapped or un-modeled environmental features, so that it may update its map before a possible collision. Simulating a full environmental interaction session is a relatively computationally intensive process but for this initial test of the VM method it is sufficiently fast. In fact, because the simulated environment is relatively small, in most instances, the simulation is actually too fast for the physical speed of the robot. The simulation can be tuned to "slow down" the physics and allow the physical robot to catch up with the simulation if need be. The full controller has 2 layers. The physical, which describes how the physical robot "follows" the virtual body. Then the virtual, which describes how one can add high level goals to the reactive nature of the virtual body.

\subsubsection{Physical Layer}

The physical robot "follows" the virtual robot through a simple proportional pose controller as follows:

$$
\vec{v}=K\left(\overrightarrow{x_{g}}-\overrightarrow{x_{c}}\right)
$$

Where $\vec{v}$ is the command velocity vector of the physical robot in the global frame, $\overrightarrow{x_{g}}$ is the global configuration of the virtual body attachment point in the real space, and $\overrightarrow{x_{c}}$ is the global current configuration of the physical robot in the real space. As distance from the virtual robot 


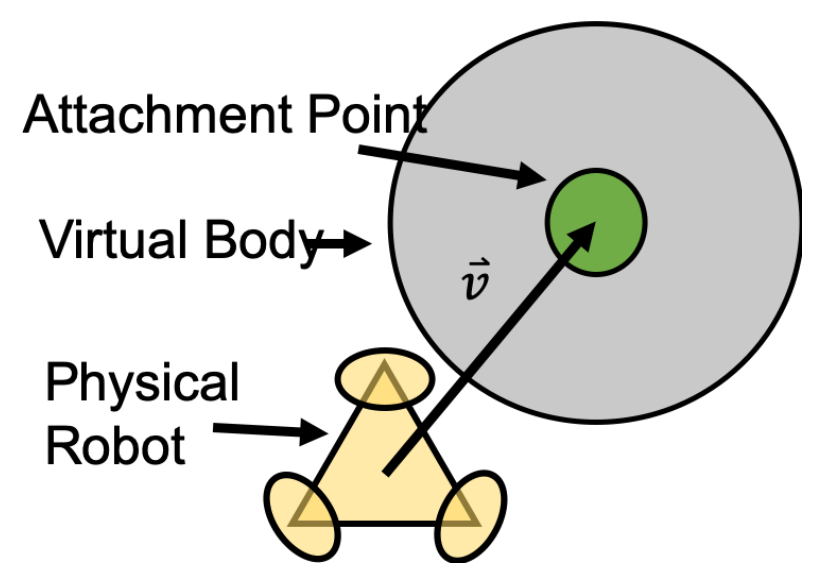

Figure 3.6: If the distance between the physical robot position and the virtual robot position increases, a command velocity is sent to the motors in the direction that will minimize this distance.

increases, or as the robot's orientation deviates from the virtual body's orientation, the controller will output a command velocity which is then sent to the robot. A diagram explaining this controller is shown in Figure 3.6. In order to improve the response of the system it is also possible to implement a full PID controller. The perfomance of this particular implementation was deemed sufficient however it would be desired for future implementations should have a more finely tuned full PID controller. The virtual body position and physical robot position is updated very frequently (around $60 \mathrm{~Hz}$ ), and because of this, the distance between the virtual body and the current robot is relatively small. This allows the physical robot to affectively mimic any particular trajectory of the virtual robot. This can lead to interesting and useful physical behaviors if the virtual mechanism is designed cleverly. One beneficial result of the physical robot "following" this trajectory blindly, is that the physical robot behavior can be altered simply through changing the virtual layer inputs. The virtual layer can be altered by changing the virtual structure, or through using a higher level path plan. 


\subsubsection{Virtual Layer}

The virtual body control is significantly more complex than the physical layer. This is because this layer has multiple inputs leading to varied complex responses. The multiple control inputs are desirable because it leads to a more flexible controller that considers a reactive component from virtual collisions to the overall path plan. In order to achieve this mixture, unlike the physical layer, this controller operates not on velocity but on forces. This is because the virtual body is inherently controlled through the LiquidFun physics engine. This means that any virtual forces imparted onto the body will cause movement of the body. In order to use higher level planning methods, additional virtual forces will be imparted onto the body in the direction of desired travel for the robot. The general control equation to include both the reactions generated by the interactions with the environment and the higher level goal is shown as follows.

$$
\overrightarrow{F_{\text {Virt }}}=\overrightarrow{F_{\text {Applied }}}+\overrightarrow{F_{\text {Env }}}
$$

$\overrightarrow{F_{V i r t}}$ is the total external force imparted onto the virtual body's center of mass, including rotational forces. $F_{\text {Applied }}$ is the force imparted by a path plan, if one is being used, in order guide the virtual body in the direction of a particular path, and $\overrightarrow{F_{E n v}}$ is the reactionary forces imparted onto the body by the virtual environment. In this way, the body can utilize the applied force as a desired direction of travel but the environment can cause deviation from an original path to ensure obstacle avoidance. A diagram of the controller's forces is presented in Figure 3.7.

The applied force can be chosen by an operator. This force can be used to impose a high level goal onto the overall motion of the system while still allowing for reactionary forces to play their part. For instance in order to set the robot in motion in a particular direction at a particular speed, 


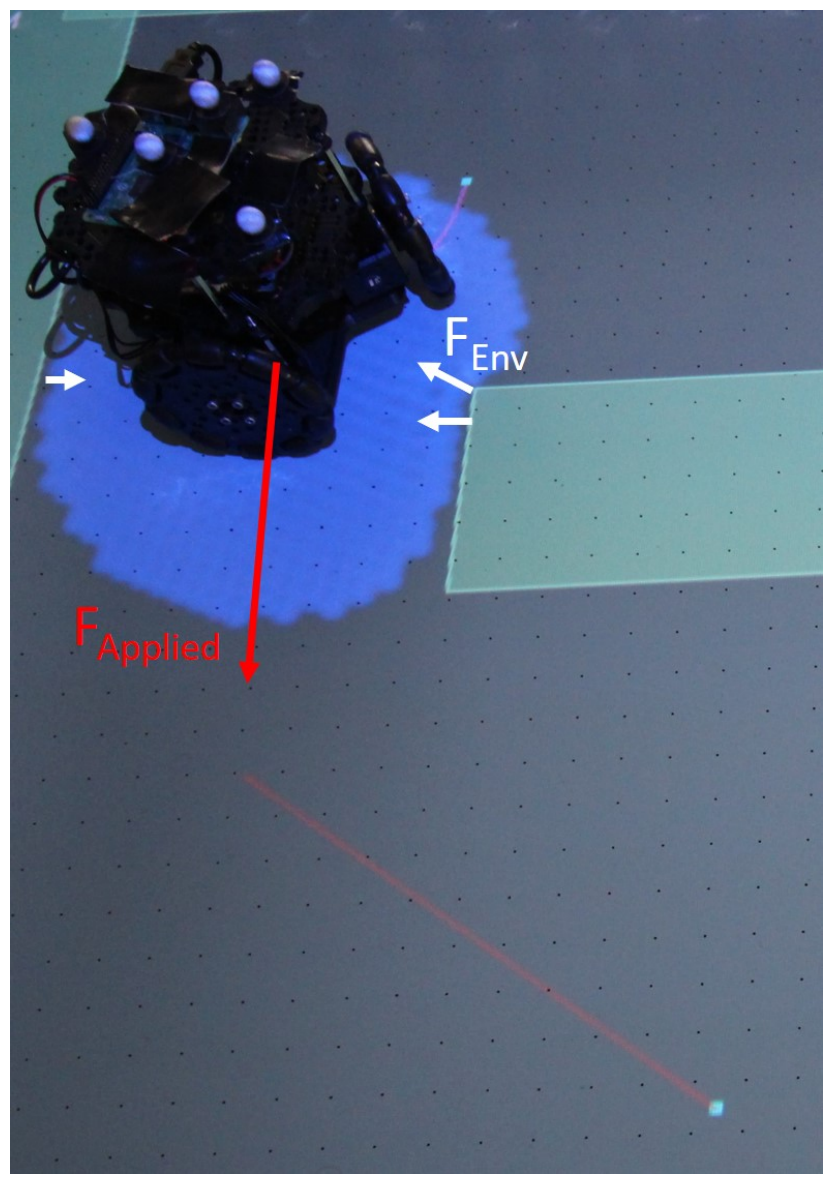

Figure 3.7: Figure showing the application of applied and environmental forces. Typically, applied forces will act through the center of mass of the virtual body while environmental forces will occur where there was contact with a virtual object. The applied force in this case is acting towards the next node PRM path plan.

such as when attempting to reach a static goal position, the equation can be chosen as follows:

$$
F_{\text {Applied }}=\frac{v_{\text {desired }}-v_{\text {current }}}{\delta t} m
$$

Using this equation in steady state without the presence of environmental disturbances has the particular benefit of settling to a desired velocity ( $\left.v_{\text {desired }}\right)$. The choice of $m$ and $\delta t$ are up to the designer but in this implementation $m$ is set as the mass of the virtual body for continuity and $\delta t$ is set as a constant value of ".1"; they can be manually tuned as necessary. Another clear choice for 
$\delta t$ would be the time difference between iterations of the physics solver.

This applied force can also be set in the form of virtual motors on joints to provide torques to rotary virtual components. LiquidFun handles motors differently than applied forces on a body so the proper way to apply motion to a joint containing a motor is by enabling the motor on the joint, setting the maximum possible torque, and set the angular speed of the motor to a desired value. The motor will rotate the body at that speed but if acted on by an external reaction the motor will resist up to the maximum torque limit and will then cap its ability to "push" back against the external force. An example of this is shown in Figure 3.8.

These, however, only describe the external forces imparted to the virtual body, another set of forces are those internal to the designed virtual mechanism. The benefit of using the physics engine with passively moving virtual mechanisms is, if cleverly designed, these internal forces can achieve desired motions without explicitly setting their values. In the same manner of physical morphology, the external virtual force will cause the internal virtual forces to produce interesting motions that can be taken advantage of. The internal forces are set and managed through LiquidFun, mostly through the use of joints and motors and do not need to be explicitly set by the user in any other way unless a particular response is desired. 


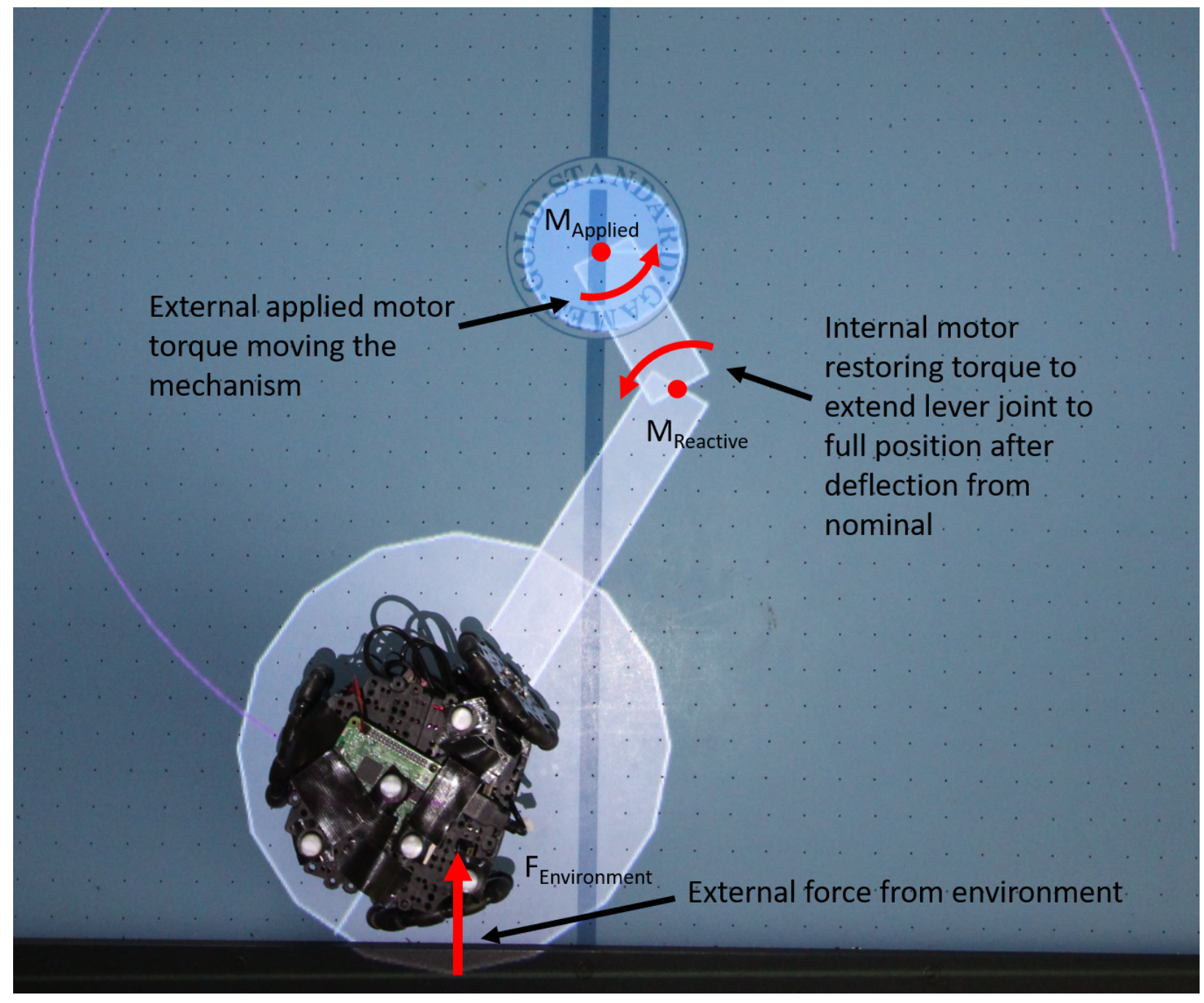

Figure 3.8: Figure showing example of internal and external forces generated in virtual mechanism. 


\section{CHAPTER 4 Experimental Design}

This section describes the two case studies which are used to determine how one can accomplish tasks using the features exhibited in the VM method. This chapter will continue as follows: First, each case study is broken into sub-experiments. These experiments are used to test a specific problem that could be encountered in practical implementations of the system. The sub-experiments for each case study build on each other and become increasingly more complex and difficult with each successive test. This section will both describe the experiments and explain their purpose in meeting the proposed claims of contribution of the VM method listed in the introduction. After each experiment, the results of that experiment will be described in detail as well as any extra unexpected observations.

\subsection{Experiment 1A: Obstacle Avoidance And Goal Reaching}

As can be seen in Figure 4.1, a blue, virtual body is attached to the physical robot. In this scenario, the green, virtual objects are static obstacles. The virtual body is made up of elastic particles lumped together, so, as it hits an obstacle, it deforms and rebounds off the obstacle. The robot attempts to stay in the center of the virtual particle mass and therefore, would not cross the obstacle. If the realworld environment is accurately mapped into the virtual environment, the robot would then "avoid" the obstacles because of the virtual body interaction. It is easy to alter the avoidance behavior by changing the properties of the particle mass. For instance, by changing the rigidity of the particles the virtual body would react differently after colliding with an obstacle (e.g., bouncing higher or 


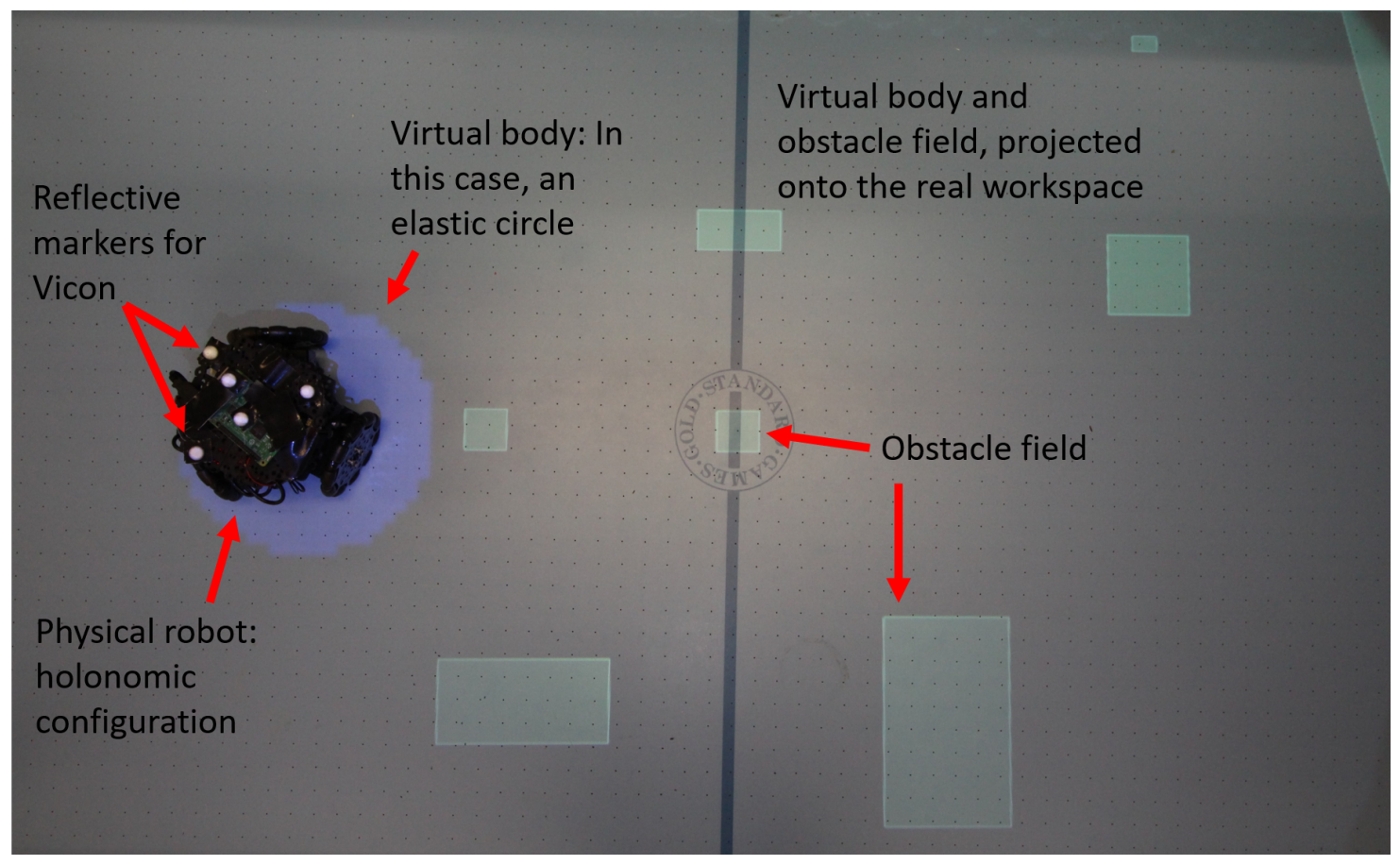

Figure 4.1: The overhead view of the virtual/physical environment. The example shown is the obstacle avoidance and goal reaching test case.

lower off the obstacle after the collision).

To show the effect of changing the virtual morphology, several iterations of a motion test through an obstacle field were conducted. In each iteration, different virtual body shapes and rigidity's were used to control the physical robot. The shapes used for the test were a circle and a triangle. Each shape was tested as a rigid body and as a soft, deformable body. That is, by adjusting parameters of the morphology, such as the shape and rigidity, one could effectively alter, or "tune", the robot behavior.

The virtual bodies were inserted into an obstacle field with the goal of making them to move from the left side to a goal in the right side of the field. The movement towards the goal is dictated by a gravitational field imparted into the system. The artificial gravity motion method was chosen for its simplicity but, movement towards the goal could have also been guided by any other 
higher-level planning algorithm. As long as the path plan is translated into forces imparted to the virtual body, the overall VM method would remain the same; this is illustrated by the methodology discussed in Figure 1.2. The trajectories generated of each virtual body are shown in Figure 4.2. It shows that, by choosing a different shape, with differing mechanical properties, one could vary the trajectories taken by the robot in this experiment. This way the trajectory generated can still utilize the passive benefits of morphology like inherent obstacle avoidance, passive action, and a robustness to mapping error, while also enjoying the benefits of a programmed method such as post-development flexibility and versatility.

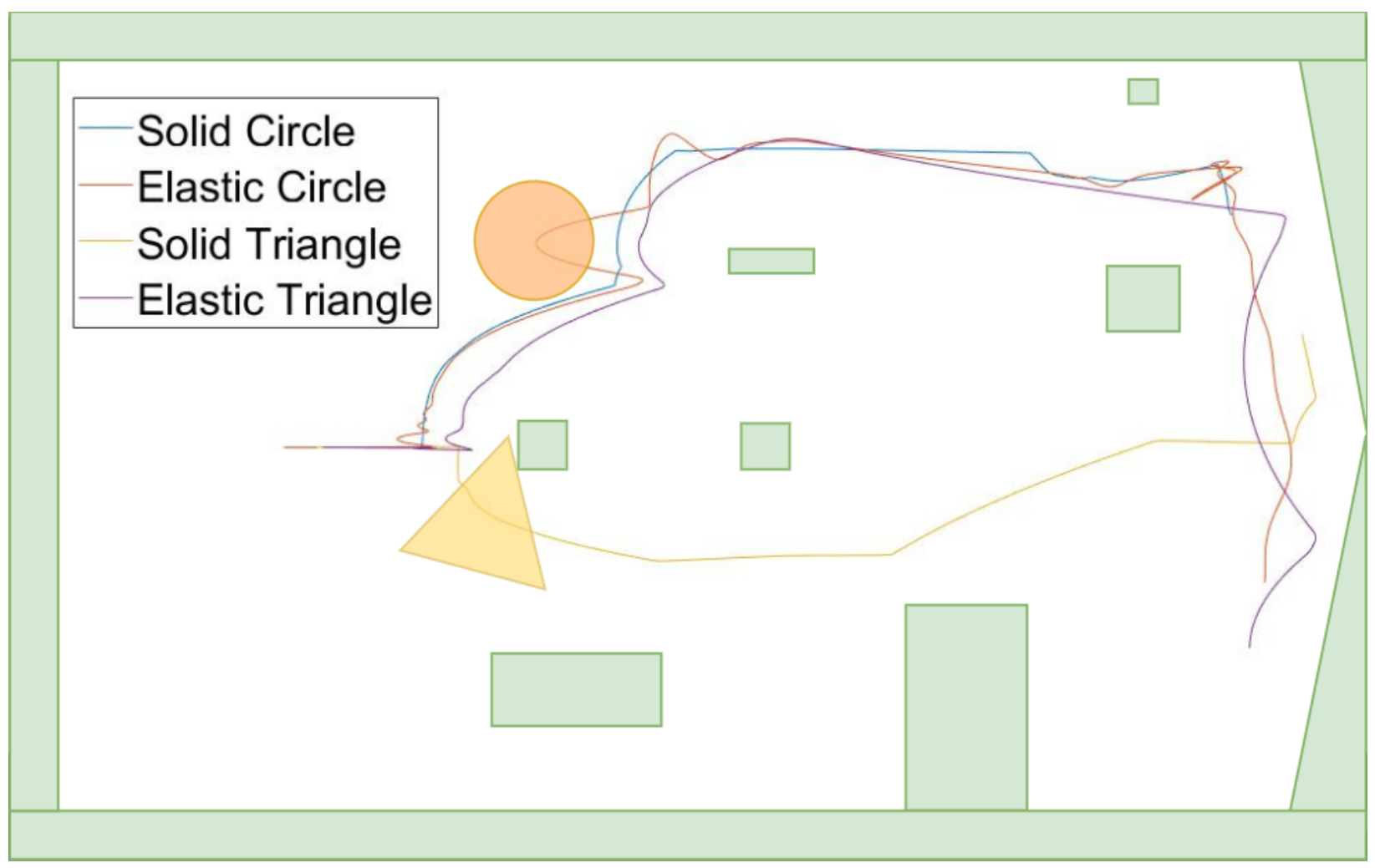

Figure 4.2: Simulation results of the obstacle avoidance and goal reaching test case. Only the simulated trajectories are shown to reduce clutter in the figure, but the physical robot trajectories follow closely. 


\subsection{Experiment 1B: Changing The Higher Level Planner}

Unfortunately, although using VM with artificial gravity to move in a particular direction demonstrated obstacle avoidance coupled with a higher level goal (i.e. moving in a particular direction), it is still subjected to many limitations when implemented in a practical situation. First, only using gravity to suggest a bulk motion of the body will result in the robot moving in a desired direction, but will not allow the robot to move to a pinpoint goal location. Secondly, this method is subjected to the classic AP problem of getting trapped in a local minima. Optimal planners can guarantee that, if a path exists to the goal, it will be found, even in the presence of minima conditions. However, optimal planners require the use of a precise and accurate map. This causes issues when applied to real world problems where one can deal with both map inconsistencies and also dynamic obstacle components. The objective of this experiment is to attempt to combine a high-level planner, which will plan a path towards the goal, with VM as a low-level layer, which will deal with all obstacle avoidance, including "sensed" map inconsistencies, unmapped obstacles, or potentially dynamic obstacles. The virtual body will serve as the obstacle avoidance layer, while the high level planner is used to reach a goal location.

A probability road map (PRM) described in [Lynch and Park, 2017] was chosen as the planner. The path plan will be solved in such a way that the shortest path to the goal from every point is known. In this manner, if the original chosen path is unable to be followed or is largely deviated from due to the presence of environmental forces, the robot just needs to find the next feasible path in order to continue towards the goal. This creates alternative routes if the desired path for whatever reason was untraversable for instance, in the event of an un-mapped obstacle blocking the route. This is not the only method possible to be implemented but it was sufficient for this experiment and desirable because of its ability to generate alternative routes towards the goal. It is important 
to note that the planning algorithm was altered so that if the robot is "stopped" for a period of time without being at the goal location, it will pick a new path to attempt as it assumes the current path is not traversable for whatever reason. This reason could be from an un-modeled obstacle or from the path being too narrow for a particular virtual body shape, etc. The bright green graph in Figure 4.3 depicts the PRM map. The light red line shows the chosen path of the connected PRM nodes and the pink line shows the actual trajectory taken by the robot.

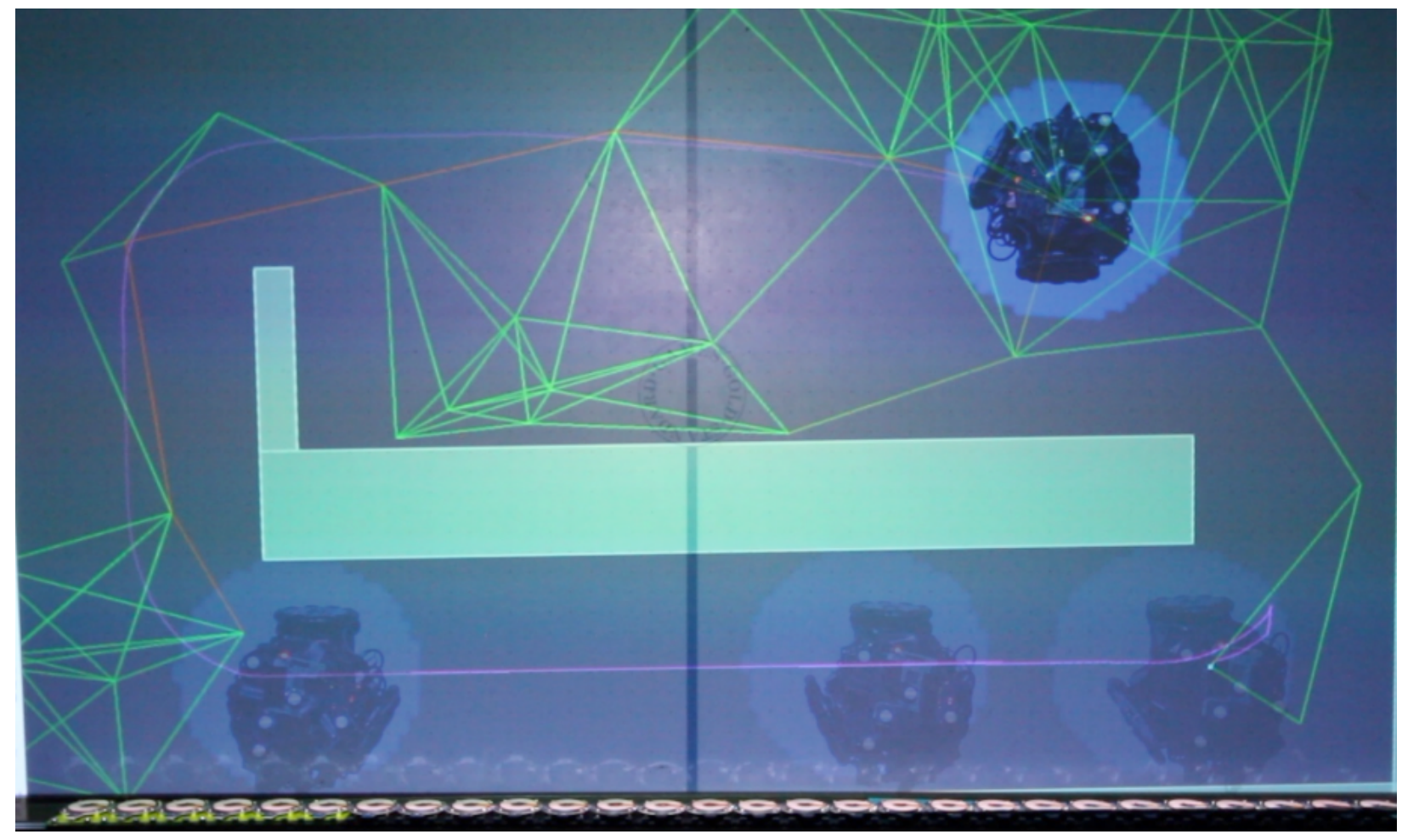

Figure 4.3: Simulation results of the PRM method implemented with a solid virtual body. The goal location is the top right of the figure and the starting location is the bottom right. The same PRM graph was used for every experiment in case study 1 . The rigid body was unable to move through the narrow corridor on the right side of the figure.

The experiment was performed with two separate virtual bodies of the same size and shape, but differing elasticity's. The environment consisted of a long L shaped obstacle, wall boundary obstacles, and the virtual body. The environment was selected as it allowed for two feasible paths towards the goal where one path was narrower than the other. This alternative path was still 


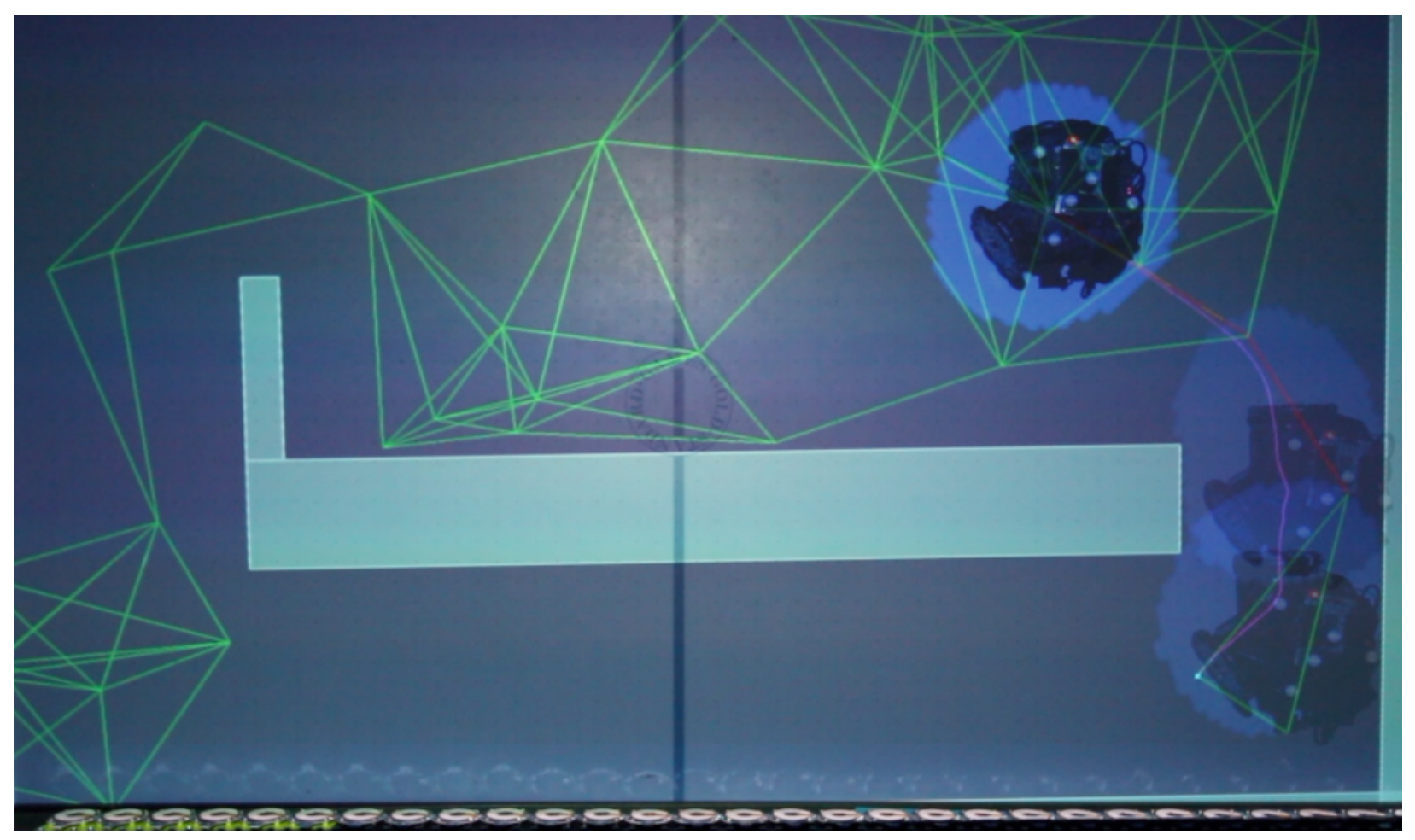

Figure 4.4: Simulation results of the PRM path plan with an elastic virtual body

traversable by the physical robot, but allowed for a shorter overall path to goal. This environment was specifically designed to demonstrate that occasionally, a flexible obstacle avoidance constraint can be beneficial as long as it still allowed for safe traversal through an obstacle section. This is desirable as opposed to designing a hard distance limit from which no obstacle can enter around the robot. It allows the design of contextual obstacle avoidance where, if necessary, the robot is willing to be closer to a wall than typical so as to provide more fluent or beneficial motion.

One observation is that an elastic virtual body was able to utilize the shorter path shown in Figure 4.4, while the rigid body needed to take the long path as it was not compliant enough to fit through the narrow gap. This also demonstrates the usefulness of including the addition of a more rich virtual embodiment as opposed to other AP methods. Simply by changing one parameter of the virtual body, the behavior was altered in such a way that the path was shortened drastically. 
However this result should be taken with a grain of salt as it was specifically designed to demonstrate this principle. It is also demonstrated, as can be seen from the trajectory map by the pink travel line, that the robot altered its path from the original PRM node path due to virtual reaction forces imparted from the obstacle. This demonstrates the efficacy of the low level reactive layer implementation of VM.

\subsection{Experiment 1C: Adding Un-mapped Obstacle}

In this particular experiment, the objective of the robot will be to move to a goal location using a high level planner while having access to a physical map that has only partial knowledge of the present obstacles. The planner may only have access to an incomplete map, but it is assumed that the "virtual world" will have complete knowledge of all the obstacles (VM requires at least knowledge of the local obstacles). This is deemed a sufficient assumption because in a real-world implementation of this experiment, a local sensor would update the virtual world of any "sensed" local obstacle in order to properly compute a movement vector. However, the only physics computation that is necessary in this experiment is the calculations of the forces against the virtual body, meaning that only the local obstacles matter for the calculation anyway. Having all obstacles pre-mapped into the virtual world just simplifies the work that would be necessary to send locally "sensed" obstacles back to LiquidFun, from the turtlebot, to put into the simulation.

The result shown in Figure 4.5 shows the path deviation caused from a static un-mapped obstacle. Essentially the light blue circular obstacle in the figure was left out of the map that generated the PRM graph. The result of this is a path that travels directly through the obstacle shown in light red in the figure. As can be seen from the pink trajectory, the VM reactive layer deviates from its path because of virtual reaction forces pushing on the virtual body from the virtual obstacle. 


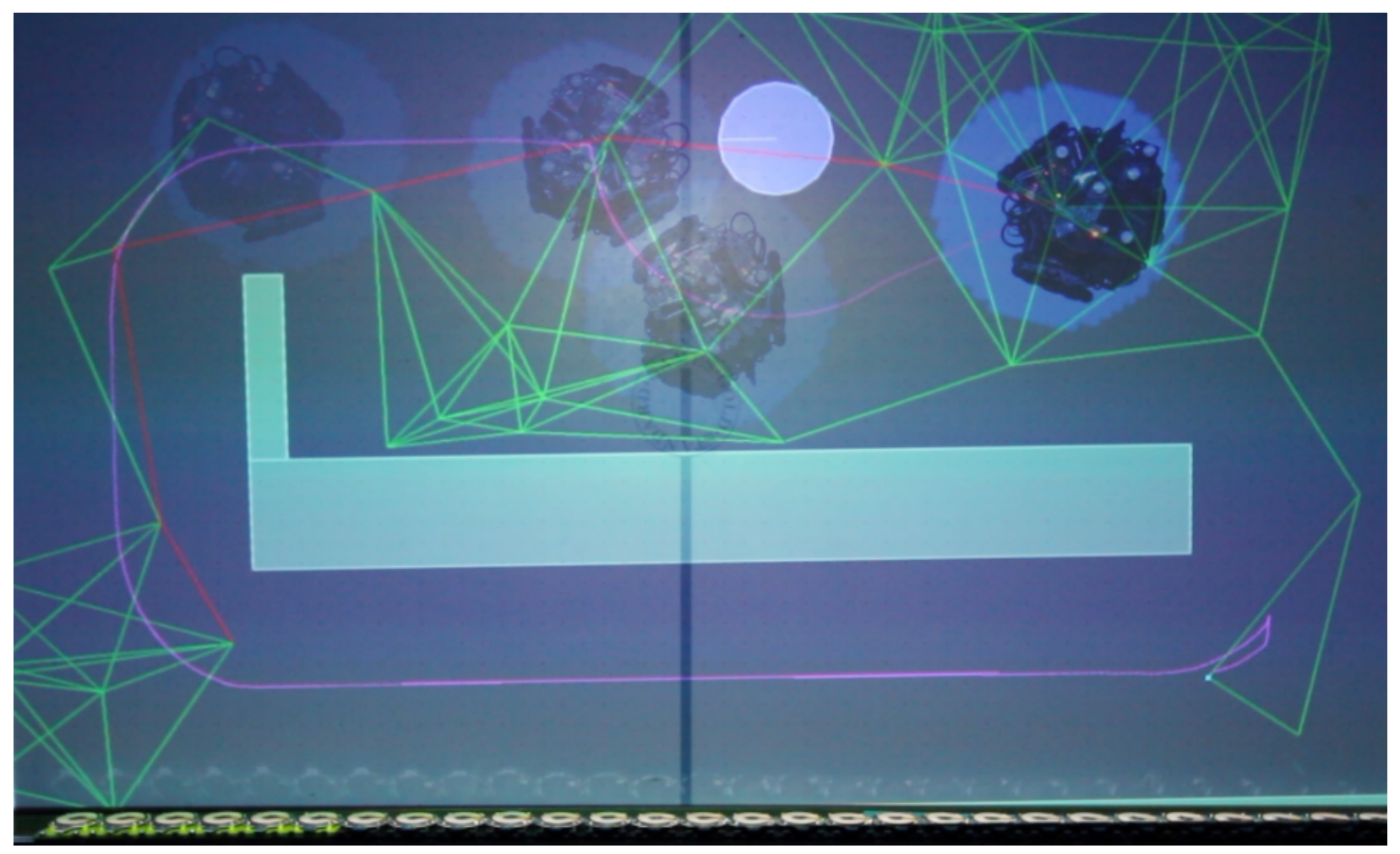

Figure 4.5: Simulation results of the PRM path plan with a rigid virtual body and an un-mapped obstacle. The un-mapped obstacle is shown in light blue in the middle of the figure.

In a practical implementation the dynamic obstacles and the un-mapped static obstacles would be added to the virtual world in the same manner. They would each be added to the virtual world and treated as kinematic bodies. At every step, the position of the "sensed" obstacles, dynamic or static, would be updated to their new positions as indicated by the local sensing method of the physical robot.

\subsection{Experiment 2A: Orbiting Object With Fixed Circular Path}

Case study 1 (Experiments 1A-1C) was able to demonstrate the implementation of VM as a low level layer in a planning system. This was able to show AP-based obstacle avoidance integrated into a high level plan. While the morphology of the virtual body is important in those experiments, it is 
only partially taken advantage of. That level of "compliance" could have also been generated using an APF method by changing the potential function of the obstacles to allow for more compliant paths. Case study 2 (Experiments $2 \mathrm{~A}-2 \mathrm{C}$ ) was developed to test more of the mechanism generation aspect of VM, because virtual embodiment is an essential principle of VM. The task of case study 2 is to orbit an object of interest in the environment. This is a rather common task in robotics as it is often desirable for mapping and perception tasks to get a more complete view of an environment or an object by perceiving it from multiple angles. In order to meet this objective using VM, one can conveniently generate a mechanism instead of explicitly determining a path. In this case, orbiting the object using a circular trajectory is sufficient. This trajectory can be generated by a combination of a lever, two gears, and a motor. The physical robot attachment point to the virtual mechanism is located on the center of the outer gear. A visualization of the virtual structure is shown in Figure 4.6.

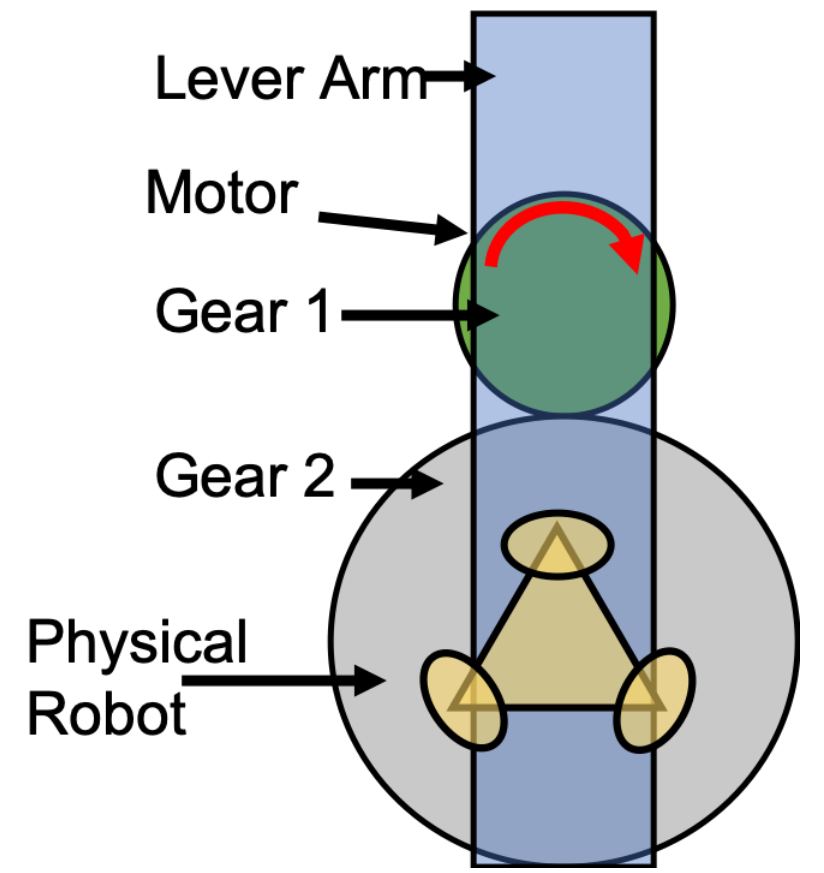

Figure 4.6: Visual representation of the designed orbiting mechanism

The physical robot while following the virtual mechanism was able to complete a trajectory around the object. This was a promising result but limited in terms of its practicality. The further 
sub experiments show improvements to the mechanism that improve the overall robustness of the method to orbit objects in a more general application. Figure 4.7 shows the trajectory generated from experiment $2 \mathrm{~A}$.

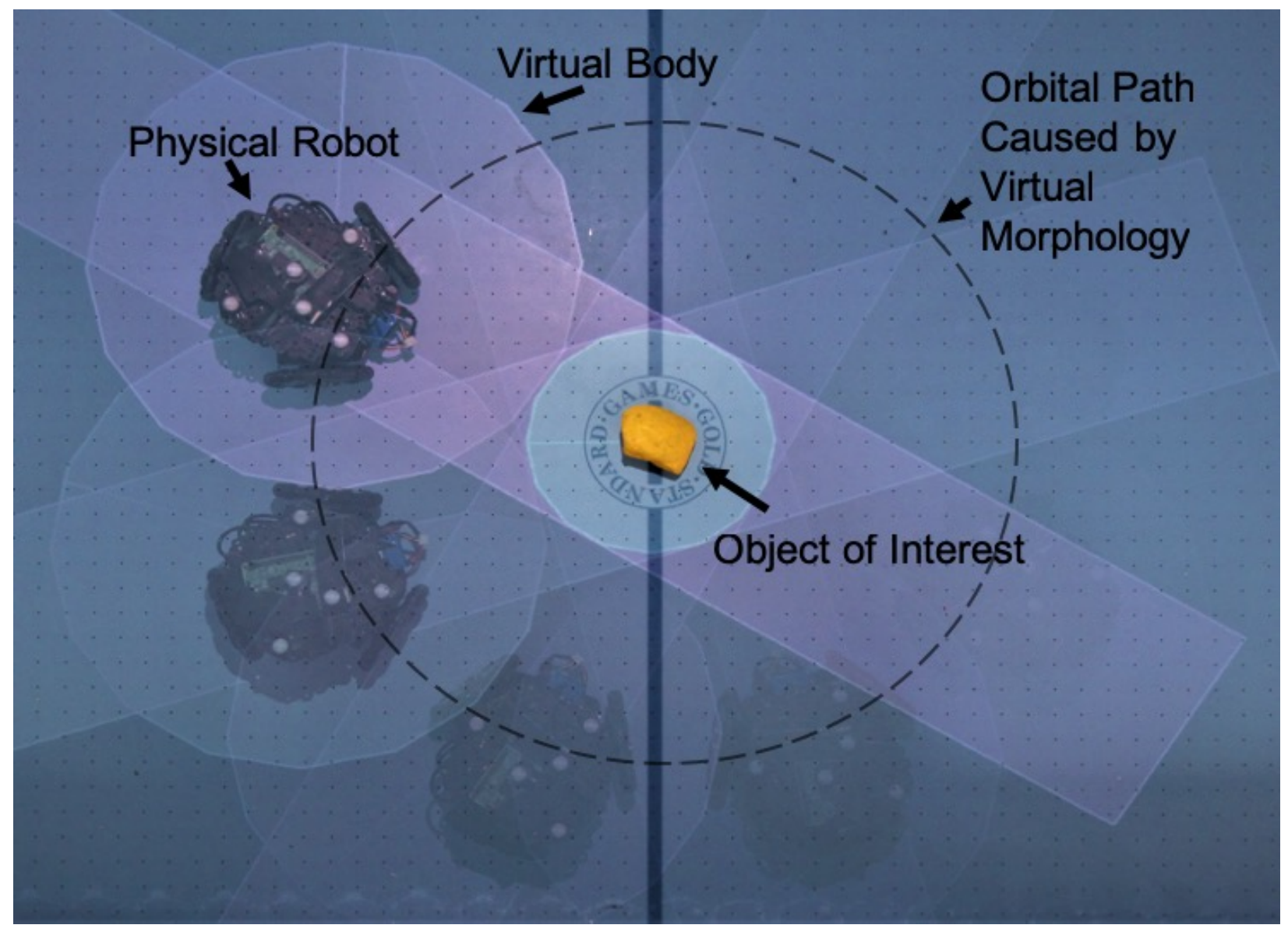

Figure 4.7: Orbit of an object using a virtual mechanism to produce trajectory

\subsection{Experiment 2B: Dealing With Obstacles While Orbiting}

The behavior from section 4.4 demonstrates orbiting capability but may experience issues if the environment is altered to contain obstacles. It is realistic to assume that there may be obstacles in the form of walls or other smaller obstacles near the object that is desired for inspection. While the previous mechanism in Figure 4.7 would not necessarily fail in such a case, it may halt its orbiting 


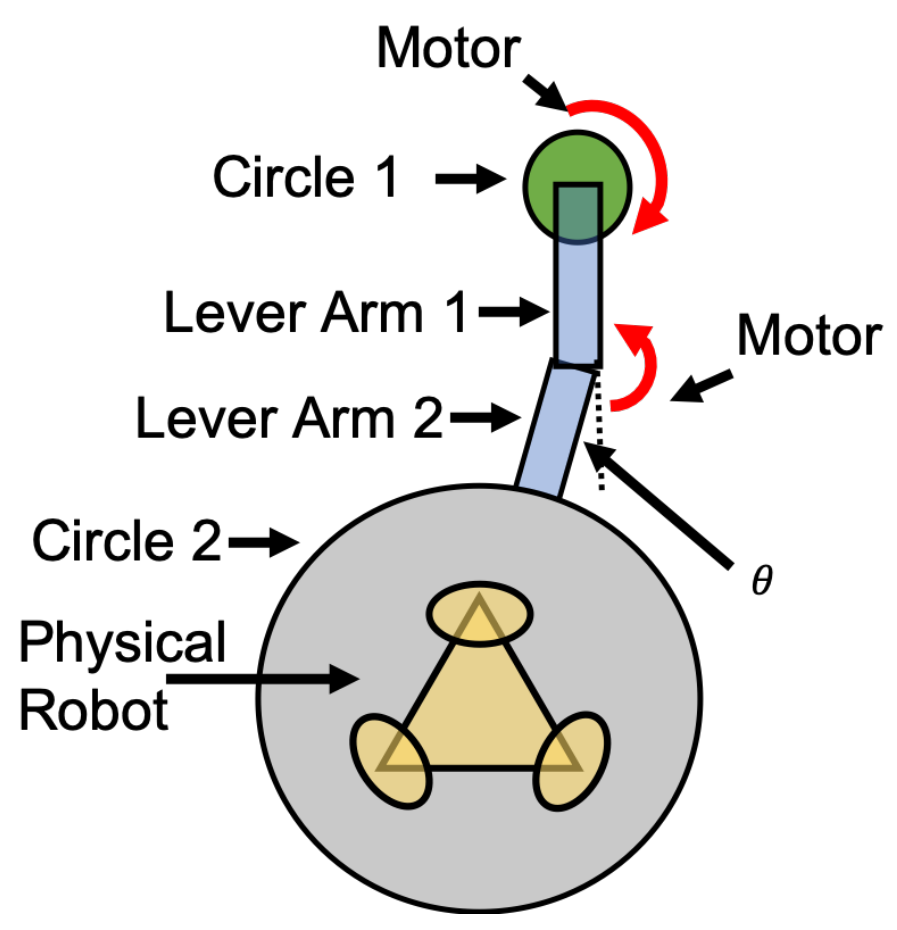

Figure 4.8: Compliant mechanism designed to handle obstacles presented to the orbit path.

trajectory. Failure, in this case, meaning if the physical robot collided with an obstacle or if the virtual mechanism broke down. By changing the virtual mechanism it is possible to change the compliance of the system in the presence of obstacles. In an attempt to observe if this halting issue can be alleviated, the mechanism was changed. This time the mechanism was made with two lever arms, two circular bodies, and two motors, as shown in Figure 4.8. The attachment point of the physical robot to the virtual mechanism is on the center of the outer circular body. The lever arms were made long enough to intentionally collide with the world boundary in an attempt to see the behavior when obstacles are present in the path of the robot, shown in Figure 4.9. The motors were each handled slightly differently. The motor attached to lever 1 was chosen to have a maximum output torque and a constant angular velocity. The motor attached to lever 2 was chosen to have a restoring torque in an attempt to simulate a torque spring. The equation dictating the restoring torque is shown below: 


$$
m_{\text {speed }}=- \text { gain } * \theta
$$

As stated previously, setting the motor torque is not done directly in LiquidFun. One sets the motor speed and a maximum torque and LiquidFun handles the rest. The gain is a tunable value. The error angle, $\theta$ is defined as the compliment to the angle between the first and second lever bar. In other words when the lever bar is straight the angle error is equal to zero.

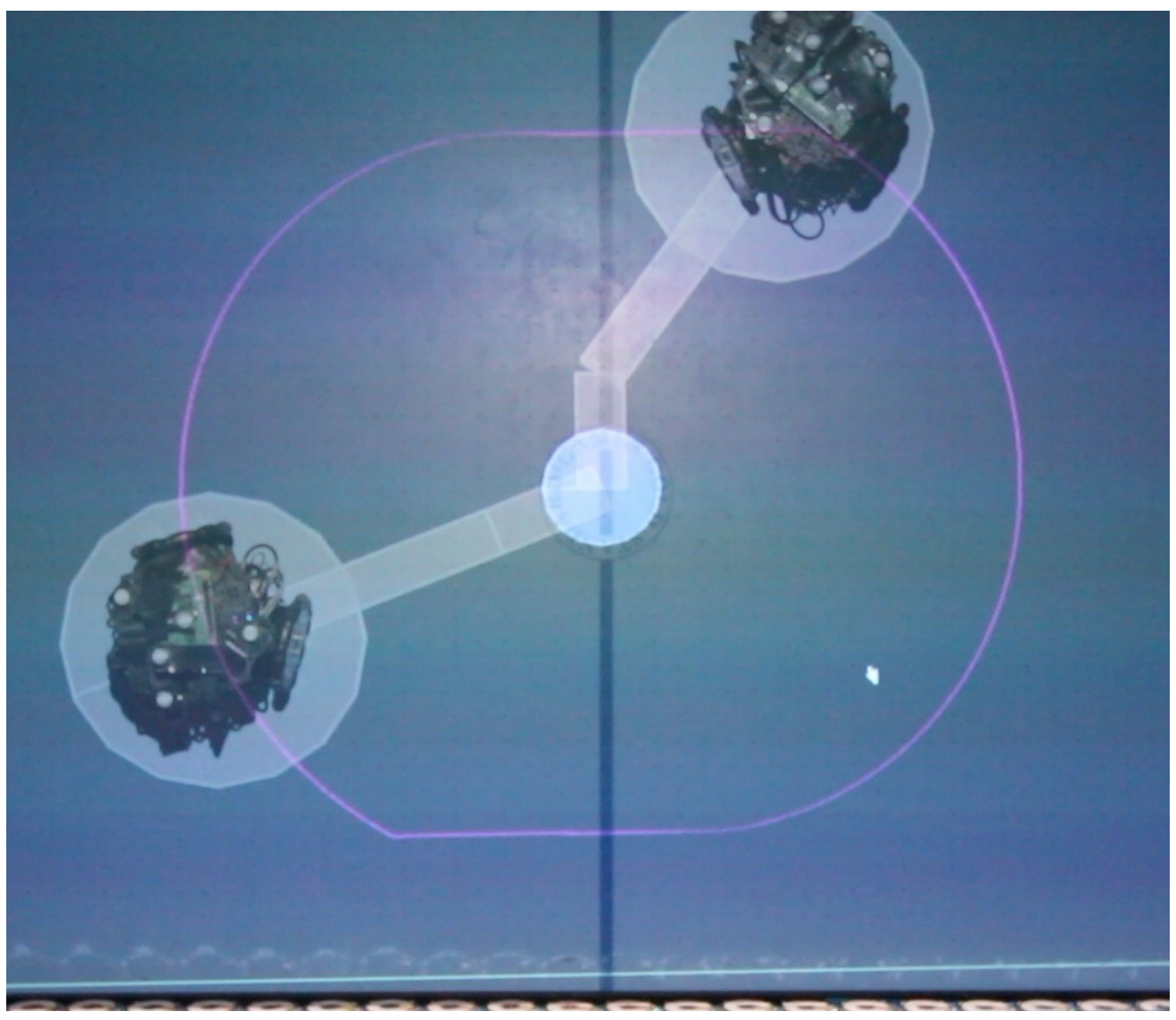

Figure 4.9: The flexibility of the lever joint allows for compliance in the presence of obstacles, in this case the virtual structure collides with the world boundary and therefore bends to continue its orbit.

In Figure 4.9 the trajectory of the end of the virtual mechanism is shown in pink. It is clear that the path the physical robot will take has been altered to be more of a squished circle rather than 
a full circle, but it is also noted that the path is continuous. This shows that even in the presence of a barrier, the mechanism still allows for compliant orbiting while also keeping the physical robot from colliding with the barrier.

\subsection{Experiment 2C: Adding Motion To The Orbited Object}

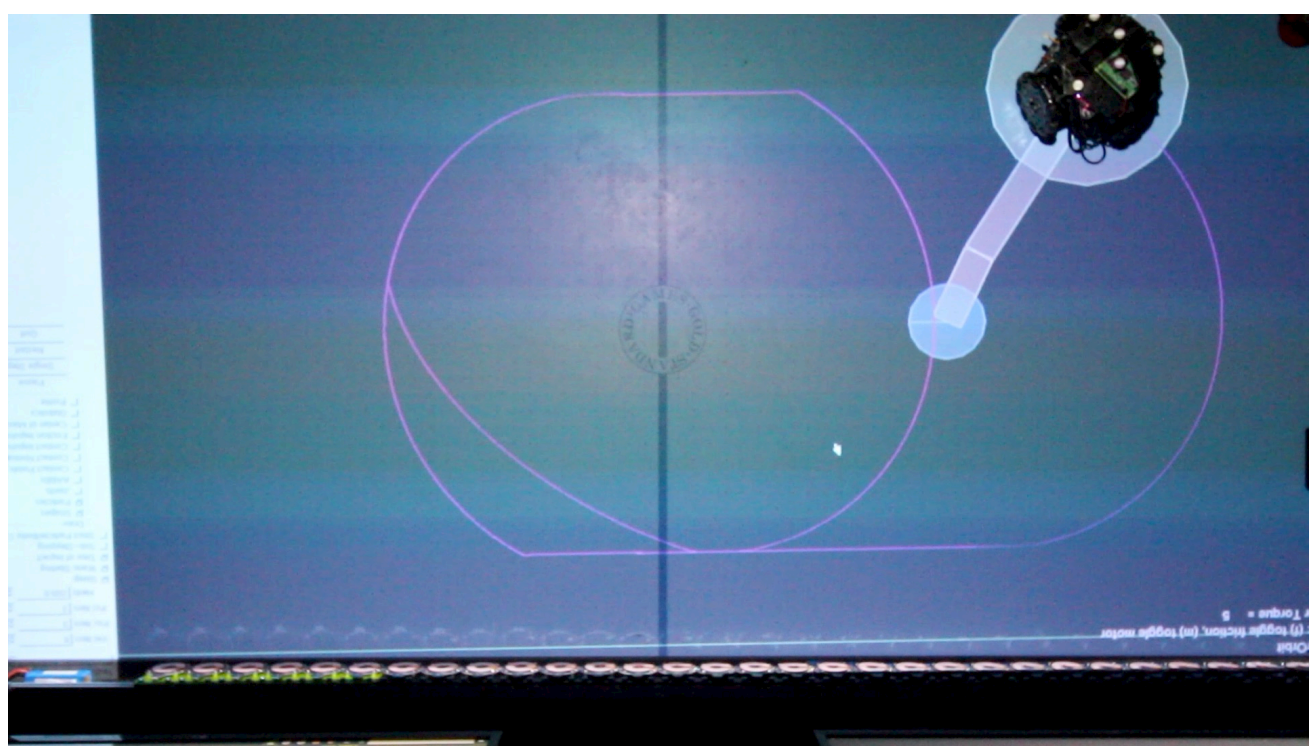

Figure 4.10: A demonstration that the orbit does not need to be about a stationary object

In the real world, the object to be orbited may not be stationary. In an attempt to observe the trajectory reaction if the object is moving, it was implemented with the previous mechanism from section 4.5. In order to simulate the movement, a virtual kinematic body was created as the central object instead of a static point. In a practical implementation of VM the robot would need to obtain a "target lock" on the desired object to be orbited. Otherwise this extended behavior could not be achieved. In this simplified demonstration, the robot has perfect knowledge of the objects location at any given point in time. In a practical implementation the object's position would be updated periodically with the "sensed" position of the object, which could possibly lead to trajectory degradation if the sensing method is noisy. 
As is seen in Figure 4.10 in pink, the trajectory moves along with the motion of the object. In this particular case the object is virtual and so is a simulation of a perfect "target lock". In practice the trajectory would most likely not look this smooth, but a similar result would be expected. 


\section{CHAPTER 5 Discussion}

\subsection{Case Study 1: Obstacle Avoidance and High level Goal Reaching}

The results of the case studies and sub-experiments led to several interesting observations. First, from experiment $1 \mathrm{~A}$, it is shown that, if designed properly, the virtual body will create a "barrier" so that the physical robot won't drive into any obstacle. It is also observed that this virtual body is "tunable" to achieve different reactions after bouncing off of an obstacle. It is important to note that this tuning is not trivial or arbitrary. If not tuned properly, the physical body could collide with an obstacle or could travel much longer distances than necessary. Therefore careful design consideration is still needed when designing this virtual morphology. The benefit, however, lies in the fact that the virtual bodies can be quickly and easily changed to obtain different behaviors, trajectories, and safety "zones" (i.e., larger virtual body, the robot will stay further away from obstacles, leading to smaller chance of collision). This quick and programmable virtual body change implies that an optimization of the virtual body can be performed to achieve better than average results in a particular environment.

From experiment 1B, it is observed that one may add a high level plan while still utilizing the reactionary nature of the VM method as a low level behavior. A benefit of the VM method is that one can implement this plan in the task space without the need for the configuration space, because reactionary forces on the virtual robot will keep the physical robot from getting close to obstacles. One should still take care, however, because without a defined configuration space it is possible to generate paths that will not be feasible that the robot will need determine how to 
neglect; As in the case of the solid virtual body example where a secondary path was chosen that went the long way around the obstacle. It was also observed that a change in virtual body shape added the possibility of traversing different paths. This helped speed "time-to-goal" performance but also required more compliance in the virtual body. This is an interesting result because it shows that, in certain scenarios, flexible constraints can be more beneficial than hard constraints if those hard constraints were not carefully decided on. This occurs frequently in robotics design where a designer knows that there is a certain "safe" area around the robot which obstacles should not be inside but how much of a safe zone is actually required for safe operation? If the safe zone is too big than the robot may not be able to traverse in narrow areas; even though, physically, it is perfectly capable of fitting, such as when traveling through doorways and thresholds. By adding in a slightly flexible constraint, such as an elastic virtual body the VM computes some more contextual insight to the problem. In general, a flexible constraint will still rest in an area where there are no obstacles within its "safe" zone but if necessary, it can slide through narrow zones if the driving force through is large enough. This still needs to be tuned however, because if the flexible constraint is made too flexible then it could cause a possible collision with an object and the physical robot. A different method of obstacle avoidance that adds a similar flexibility is that explored by [Borenstein and Koren, 1991] who uses vector field histograms which can handle obstacle cluttered environments and handles sensor inconsistencies, readily.

Experiment $1 \mathrm{C}$ showed VM has the desirable benefit of readily handling map inconsistencies. If an obstacle was not considered when generating the global map, as long as local sensors can map it into the virtual space in time for the virtual collision, the virtual body will glance off of the obstacle and continue on its path. Unfortunately, when implementing a method that uses a global map that does not consider the robot configuration, or only has a loose representation of obstacles, careful design and thought is required when considering the overall planning algorithm (also discussed from experiment 1B). So while VM allows for more flexibility and for quick implemen- 
tation without worrying about obstacle collision, it also allows for infeasible paths to be generated and considered which may slow down the overall goal of arriving at the goal location. Therefore, it is imperative that an efficient online re-planning algorithm is mixed in to ensure sufficient results if the first plan turns out to be a failed path.

Another lesson learned from experiment $1 \mathrm{C}$ is from dynamic obstacles. There is a potential for collision with dynamic obstacles since the physical robot lags the virtual body's motion. If there is a head on virtual collision the virtual body will be pushed back, but the physical robot will need to slow down, stop, and speed up in the opposite direction. If the dynamic obstacle is fast enough it may run into the physical robot before it has time to evade. One potential solution to alleviate this issue is by using different virtual body shapes to "deflect" and move to the side of the obstacle instead of directly backwards, where the robot would need to fully reverse its current trajectory in order to catch back up to its virtual counterpart. The problem could also be alleviated by tuning the physical controller layer to have a faster response.

\subsection{Case Study 2: Orbiting Mechanism}

From this case study, it was observed that it is possible to generate continuous orbits and paths. These paths are commonly used in perception tasks for robotics, where observing an object or area from multiple angles provides better contextual information about the environment. The first virtual mechanism created allowed the physical robot to perform an orbit around an object. The mechanism was created from two gears and a lever bar and was rather complex. It worked well for its given task until the environment was altered. The environment was altered by adding virtual barriers at the world boundaries. When the virtual mechanism interacted with the barriers a virtual reactionary force was generated such that it halted the motion of the orbit. This could be considered beneficial 
because, instead of continuing its orbit and running into the boundary, it stopped motion. It acted almost as a fail safe, only continuing when the obstacle is removed. The reactionary component of VM helps to add an extra obstacle avoidance behavior to the path trajectory. In the second experiment it was shown that this behavior can be enhanced simply by a virtual mechanism change. Not only can you achieve obstacle avoidance and circular path following, but also can add compliant behavior that will allow the robot to continue on its path even in the presence of obstacles. 


\section{CHAPTER 6 Conclusions}

From the work conducted in this project it was shown that the problem statement was met in the following ways:

1. Determine which, if any, of the principles of physical morphology can be utilized virtually to enable behavioral control of the robot:

This was met by an investigation of background literature. The discovered principles were: embodiment enables complex analog "computations" to occur naturally; physics is the driving force in this analog computation; and, in order to enable this embodied computation, physics must drive the interactions of the embodiment and its environment. These principles were emulated virtually, by creating a virtual world with artificial physics and a virtual embodiment to interact with that virtual world.

2. Build a system to demonstrate and implement the blending of those principles with digital methods:

This was accomplished through the methodical creation of the VM method in a physical and demonstrative system. It was shown to blend with digital methods by it's implementation in a layered path plan as shown in case study 1 of the experimental section.

3. Explore the design of several use-case experiments of this method to determine its efficacy to solve a given problem:

This was performed through the experimental design and discussion of the observed results. 
Several limitations were also learned from conducting this research. The available behaviors are limited through the limitations of the physics simulator being used. For instance, when using an elastic body in LiquidFun, one cannot obtain an angular position of the virtual body because LiquidFun only calculates this for rigid bodies. Another limitation is through the users creativity. This is why study of mechanism design is imperative if this method is to be used, because the ability to create mechanisms will directly affect the behaviors that are able to be generated. It was also discovered, through experiment $1 \mathrm{C}$ that fast moving dynamic obstacles are rather troublesome as they can cause potential collisions that need to be taken into account. While it was not an issue in these small scale experiments, it is also still suspected that the computational cost of this method could also cause significant issues in larger systems where quick responses are important. Some methods of dealing with these issues were discussed briefly in the thesis but future exploration of this method will work on dealing with these issues and more.

Some other directions of future work will be to determine methods for automated virtual mechanism generation, improvement of planning and re-planning algorithms to be implemented in VM's high level planner, utilization of multi-robot collaborative systems to further test the benefits of morphologic computation systems, and also determining methods of more fluidly traversing the space between physical morphological computation and programming/ virtual morphological methods (i.e. taking a discovered mechanism from virtual morphology and creating a physical morphology based off of the virtual and vice-versa). 


\section{Bibliography}

[Liq, 2014] (2014). Liquidfun. https://github.com/google/liquidfun.

[Tur, 2019] (2019). Turtlebot3. https://github.com/ROBOTIS-GIT/turtlebot3.

[Akkaya et al., 2019] Akkaya, I., Andrychowicz, M., Chociej, M., Litwin, M., McGrew, B., Petron, A., Paino, A., Plappert, M., Powell, G., Ribas, R., et al. (2019). Solving rubik’s cube with a robot hand. arXiv preprint arXiv:1910.07113.

[Amend et al., 2012] Amend, J. R., Brown, E., Rodenberg, N., Jaeger, H. M., and Lipson, H. (2012). A positive pressure universal gripper based on the jamming of granular material. IEEE Transactions on Robotics, 28(2):341-350.

[Apker and Potter, 2012] Apker, T. B. and Potter, M. A. (2012). Robotic swarms as solids, liquids and gasses. In 2012 AAAI Fall Symposium Series.

[Borenstein and Koren, 1991] Borenstein, J. and Koren, Y. (1991). The vector field histogram-fast obstacle avoidance for mobile robots. IEEE transactions on robotics and automation, 7(3):278288.

[Cheney et al., 2014] Cheney, N., MacCurdy, R., Clune, J., and Lipson, H. (2014). Unshackling evolution: evolving soft robots with multiple materials and a powerful generative encoding. ACM SIGEVOlution, 7(1):11-23.

[Greiner, 1990] Greiner, H. (1990). Passive and active grasping with a prehensile robot endeffector. Technical report, MASSACHUSETTS INST OF TECH CAMBRIDGE ARTIFICIAL INTELLIGENCE LAB. 
[Gu et al., 2018] Gu, Y., Ohi, N., Lassak, K., Strader, J., Kogan, L., Hypes, A., Harper, S., Hu, B., Gramlich, M., Kavi, R., et al. (2018). Cataglyphis: An autonomous sample return rover. Journal of Field Robotics, 35(2):248-274.

[Ha, 2018] Ha, D. (2018). Reinforcement learning for improving agent design. arXiv preprint arXiv:1810.03779.

[Kapitanyuk et al., 2017] Kapitanyuk, Y. A., Proskurnikov, A. V., and Cao, M. (2017). A guiding vector-field algorithm for path-following control of nonholonomic mobile robots. IEEE Transactions on Control Systems Technology, 26(4):1372-1385.

[Karaman and Frazzoli, 2011] Karaman, S. and Frazzoli, E. (2011). Sampling-based algorithms for optimal motion planning. The international journal of robotics research, 30(7):846-894.

[Khatib, 1985] Khatib, O. (1985). Real-time obstacle avoidance for manipulators and mobile robots. In Proceedings. 1985 IEEE International Conference on Robotics and Automation, volume 2, pages 500-505.

[Lee and Park, 2003] Lee, M. C. and Park, M. G. (2003). Artificial potential field based path planning for mobile robots using a virtual obstacle concept. In Proceedings 2003 IEEE/ASME International Conference on Advanced Intelligent Mechatronics (AIM 2003), volume 2, pages 735-740. IEEE.

[Lipson and Pollack, 2000] Lipson, H. and Pollack, J. B. (2000). Automatic design and manufacture of robotic lifeforms. Nature, 406(6799):974.

[Liu et al., 2018] Liu, Y., Gao, J., Liu, C., Zhao, F., and Zhao, J. (2018). Reconfigurable formation control of multi-agents using virtual linkage approach. Applied Sciences, 8(7):1109.

[Lynch and Park, 2017] Lynch, K. M. and Park, F. C. (2017). Modern Robotics: Mechanics, Planning, and Control. Cambridge University Press. 
[McGeer, 1990] McGeer, T. (1990). Passive dynamic walking. I. J. Robotic Res., 9(2):62-82.

[Müller and Hoffmann, 2017] Müller, V. C. and Hoffmann, M. (2017). What is morphological computation? on how the body contributes to cognition and control. Artificial life, 23(1):1-24.

[Pereira et al., 2016] Pereira, G. A. S., Choudhury, S., and Scherer, S. (2016). A framework for optimal repairing of vector field-based motion plans. In 2016 International Conference on Unmanned Aircraft Systems (ICUAS), pages 261-266.

[Puterman, 1990] Puterman, M. L. (1990). Markov decision processes. Handbooks in operations research and management science, 2:331-434.

[Quinlan and Khatib, 1993] Quinlan, S. and Khatib, O. (1993). Elastic bands: Connecting path planning and control. In [1993] Proceedings IEEE International Conference on Robotics and Automation, pages 802-807. IEEE.

[Sims, 1994] Sims, K. (1994). Evolving virtual creatures. In Proceedings of the 21st annual conference on Computer graphics and interactive techniques, pages 15-22. ACM.

[Spears et al., 2004] Spears, W. M., Spears, D. F., Hamann, J. C., and Heil, R. (2004). Distributed, physics-based control of swarms of vehicles. Autonomous Robots, 17(2-3):137-162.

[Stentz et al., 1995] Stentz, A. et al. (1995). The focussed $\mathrm{d}^{\wedge *}$ algorithm for real-time replanning. In IJCAI, volume 95, pages $1652-1659$.

[Warren, 1989] Warren, C. W. (1989). Global path planning using artificial potential fields. In Proceedings, 1989 International Conference on Robotics and Automation, pages 316-321. Ieee. 